\title{
Clutter Reduction and Target Tracking in
}

\section{Through-the-Wall Radar}

\author{
Hongqing Liu, Senior Member, IEEE, Chen Huang, Lu Gan, Senior Member, IEEE, \\ Yi Zhou, Member, IEEE, Trieu-Kien Truong, Life Fellow, IEEE
}

\begin{abstract}
This work addresses the problem of tracking targets behind the wall using through-the-wall radar. To that end, the wall reflection, i.e., clutter, must be eliminated first because it interferes with the subsequent image formation operation. The low-rank of the clutter and sparseness of the useful signal are utilized to devise a joint low-rank and sparse framework to simultaneously suppress the clutter and recover the target returns, where alternating direction method of multipliers (ADMM) approach is developed to solve the corresponding optimization. After that, an effective observation window scheme is proposed to detect the target and further to facilitate the tracking process. The tracking is finally provided by Kalman filter and particle filter. The numerical studies are provided to demonstrate that the performance of the joint estimation algorithm is superior to that of other methods in terms of clutter removal and tracking accuracy.
\end{abstract}

\section{Index Terms}

Through-the-wall radar, clutter reduction, tracking, particle filter, Kalman filter.

\section{INTRODUCTION}

Through-the-wall radar (TWR) systems operate from the determination of the building layout to the detection, identification, classification, and tracking of targets that are inside enclosed buildings. Because of that, TWR plays a essential role in military and civilian applications such as search and rescue missions, urban surveillance, and anti-terrorist operations [1]-[4]. In order to do so, obtaining a high-precision imaging is a necessary prerequisite for

Hongqing Liu is with Chongqing Key Lab of Mobile Communications Technology, Chongqing University of Posts and Telecommunications, Chongqing 400065, China. E-mail: hongqingliu@ outlook.com.

Chen Huang and Yi Zhou are with School of Communication and Information Engineering, Chongqing University of Posts and Telecommunications, Chongqing 400065, China. E-mail: chenhuang_cqupt@outlook.com; zhouy@cqupt.edu.cn.

Lu Gan is with the College of Engineering, Design and Physical Science, Brunel University, London UB8 3PH, U.K. E-mail: lu.gan@brunel.ac.uk.

Trieu-Kien Truong is with the Department of Information Engineering, I-Shou University, Kaohsiung County 84001, Taiwan. E-mail: truong@isu.edu.tw. 
these tasks. However, imaging indoor scenes is a challenging task due to strong front-wall reflections, which are usually stronger than the returns from the area of interest, thereby obscuring the weak indoor targets' returns. To overcome this issue, prior to image formation, front-wall reflections need to be suppressed to reveal indoor targets.

A classical method for wall reflections reduction is background subtraction [1]. However, the measurements of the exact empty scene are hard to obtain in real applications. Using subspace theory, singular value decomposition (SVD) is utilized on B-scan matrix to extract the target signatures and has been adopted as a front-wall electromagnetic (EM) return reduction for TWR sensing systems in [5]-[7]. Different from the SVD based method that requires full data, a method of clutter reduction, which uses a modulated Discrete Prolate Spheroidal Sequences (DPSS) basis to separately represent the target return, has been proposed in [8], [9]. In recent studies, two observations are revealed that the front wall reflections present a low-rank property, while the target returns present a sparse property. Therefore, the joint low-rank and sparse approaches for clutter reduction and scene reconstruction have been developed based on compressed sensing (CS) technique [10]-[17]. Particularly, in [10]-[12], Tang et al. used an iterative soft threshold method to solve the joint optimization problem, while in [15], the optimization problem was solved utilizing the mathematical toolbox TFOCS [18].

After the clutter reduction, detection and tracking tasks for moving targets in enclosed space can be conducted. In the previous TWR tracking literature, the change detection method was proposed to detect the moving targets [19], [20]. Although the it is efficient and simple in real scenarios, the target information, for example, position and speed, cannot be provided during the process.

The tracking process involves detection and estimation of targets [21]. For a pure tracking problem, many approaches have been proposed in the tracking literature, such as probabilistic data association filter (PDAF) [22], probability hypothesis density (PHD) [23], [24]. For complex motions, the interactive multiple model (IMM) method has been reported to improve the positioning accuracy in tracking [25], [26]. Unfortunately, the applications in TWR are scare because of the complex indoor environment. Kalman filter (KF) has been reported in previous studies of TWR tracking [27], [28], but it cannot deal with the data association uncertainty problem.

More importantly, most studies in TWR only focus on one part of the TWR systems such as clutter removal or imaging or tracking. In this work, a complete system starting from clutter suppression, imaging, detection, to the target tracking is designed to demonstrate the capability of the TWR system. For the clutter reduction and the image formation, an approach of joint low-rank and sparse framework is developed and studied under different situations. It is assumed that the TWR system is mounted on a ground vehicle traveling around the building. The purpose of this study is to provide insights on the effects of different types of walls and different signal-to-noise ratios to the recovery approach. Compared with the existing methods, the sparse based method suppresses the clutter well and is able to form the image scenery of the targets. In the tracking parts, a simple window detection strategy is developed 
to effectively reduce false alarms, which consists of a global search and observation window. To compensate the effect of the wall, estimated position is also compensated by the offset. Considering the uncertainty of the target motions, two dynamic models, namely constant velocity (CV) and constant acceleration (CA) models, are utilized. The IMM method is then explored to combine the estimation target states. Finally, IMM-based with Kalman filter $(\mathrm{KF})$ or Particle filter $(\mathrm{PF})$ are developed to provide tracking trajectories. The experimental results indicate that the proposed system outperforms the existing methods.

The rest of the paper is organized as follows. The TWR signal model is presented in Section II. In Section III, algorithms developments are provided where the clutter reduction, image formation, and tracking are respectively introduced. In Section IV, numerical studies for the proposed approach are presented to demonstrate the performances. Finally, this paper concludes with a brief summary in Section V.

\section{TWR SIgNAL MODEL}

In a traditional TWR system, the stepped-frequency synthesis signal, which contains a series of equi-spaced frequencies signals, is usually utilized to achieve a good range resolution [29]. In this work, however, the monofrequency signal with center frequency at $f_{c}$ is studied to demonstrate its performance. Suppose that the antenna is located at several scan positions parallel to the wall on a vehicle traveling around the building to synthesize a horizontal linear array with $N$ elements and the number of targets behind the wall is indicated by $M$.

During the process, the radar returns are measured and stored by the receiver. Let $e_{n}(t)$ denote the radar return at the $n$-th location, which can be expressed as

$$
e_{n}(t)=e_{n}^{w}(t)+e_{n}^{t a}(t)+s_{n}(t)
$$

where $t$ is the sampling time, $t=0,1, \cdots, T$, and $T$ denotes the total number of time stamps, $e_{n}^{w}(t)$ denotes the wall clutter, $e_{n}^{t a}(t)$ represents the target return, and $s_{n}(t)$ is the noise.

The scene is subdivided into orthogonal grids involving $Q$ pixels along the $x$ (crossrange) and $y$ (downrange). The signal traveling path between the $n$-th antenna location and the $q$-th pixel is depicted in Fig.1, where $l_{q, a i r 1, t}$, $l_{q, w a l l, t}$, and $l_{q, a i r 2, t}$ represent the distances traveled between transmitter antenna and $q$-th pixel in the air, in the wall, and in the air, respectively, and $l_{q, a i r 1, r}, l_{q, w a l l, r}$, and $l_{q, a i r 2, r}$ are defined the same way, but corresponding to the received antenna.

From Fig.1, the propagation delay $\tau_{n, q}$ is calculated by

$$
\begin{aligned}
\tau_{n, q}= & \frac{l_{q, a i r 1, t}+l_{q, a i r 2, t}}{c}+\frac{l_{q, a i r 1, r}+l_{q, a i r 2, r}}{c} \\
& +\frac{l_{q, w a l l, t}+l_{q, \text { wall }, r}}{v}
\end{aligned}
$$




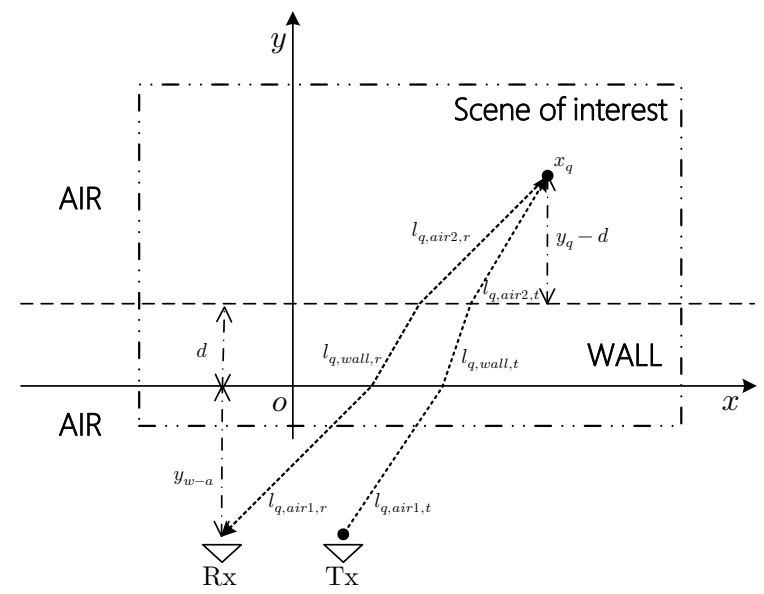

Fig. 1: Geometry for signal traveling path between the $q$-th pixel and transceiver.

where $c$ is the speed of light and $v=c / \sqrt{\epsilon_{r}}$ denotes the speed of propagation in the wall, and $\epsilon_{r}$ is the relative permittivity of the wall. Let $h_{n}$ denote the radar signal with center frequency $f_{c}$ received at the $n$-th antenna location, and it is given by

$$
h_{n}=\sum_{q=1}^{Q} \sigma_{q} \exp \left(-j 2 \pi f_{c} \tau_{n, q}\right),
$$

where $\sigma_{q}=e_{n}\left(\tau_{n, q}\right)$ is the reflection coefficient of the $q$-th pixel at the $n$-th antenna position. Based on delay-andsum beamforming, the complex amplitude of the $q$-th pixel is

$$
I(q)=\frac{1}{N} \sum_{n=1}^{N} h_{n} \exp \left(j 2 \pi f_{c} \tau_{n, q}\right) .
$$

In (4), $I(q)$ contains strong wall clutter, which overwhelms the targets of interest. Therefore, prior to image formation, the clutter needs to be reduced. Moreover, it is also imperative to track the positions of the targets all the time. In what follows, a framework for clutter suppression, image formation and targets tracking is proposed for TWR tracking system.

\section{Algorithm Developments}

In this section, the TWR tracking task is divided into two stages and its signal processing flow is provided in Fig.2. The first stage includes reduction of clutter and the image formation process. To this end, a low-rank and sparse constrained optimization model is introduced to separate the wall clutter and targets' returns. After that, an alternating direction method of multipliers (ADMM) [30] is developed to solve the optimization problem. The target components are then utilized to produce the image. In the second stage, a target location process, called target region detector, is designed to locate the targets by finding the most energetic pixel of each image. To improve 
the measurement robustness, the location error is eliminated by an offset estimation method. Based on the target measurement, the IMM-based KF or PF filters are finally devised to track the targets, respectively.

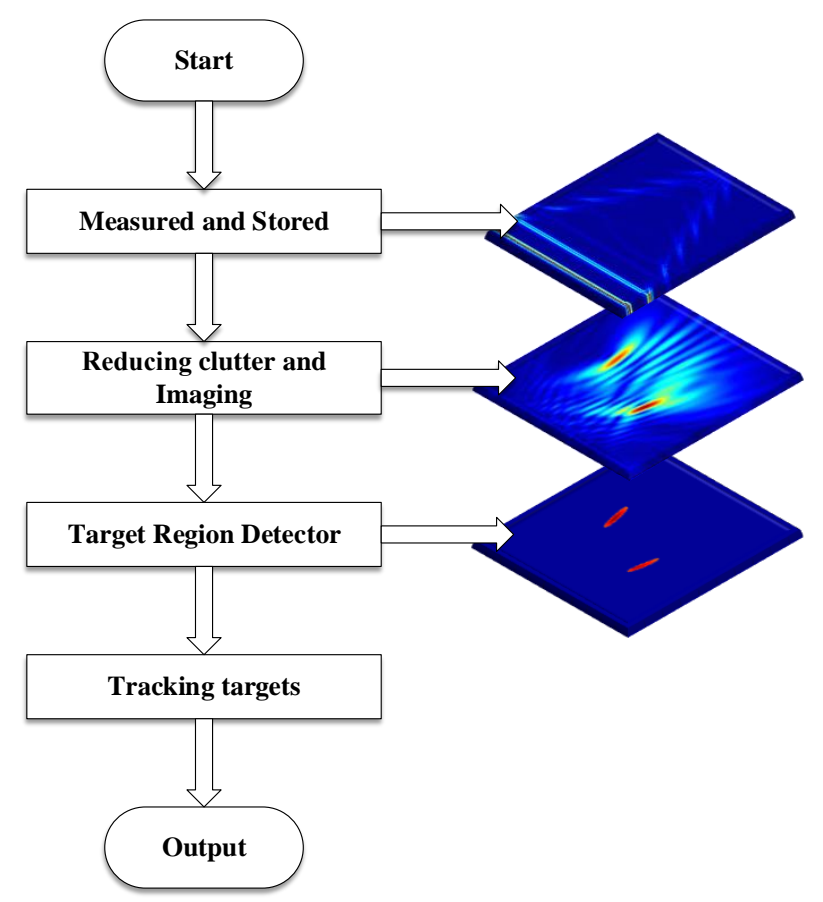

Fig. 2: Flowchart of TWR tracking system.

\section{A. Clutter suppression and image formation: Low-rank and sparse model}

Utilizing the matrix notation, the radar returns is denoted by $\mathbf{E} \in \mathbb{R}^{N \times T}$, where $T$ is the number of time samples for $e_{n}(t)$. Since the antenna positions and the wall are parallel, each column of the wall reflection matrix $\mathbf{E}^{w}$ is (approximately) equal, i.e., $\mathbf{E}^{w}$ is a low-rank matrix. For the scene of interest, the target occupies only a small area. Thus, most elements of $\mathbf{E}^{t a}$ are zeros. Using matrix notations again, (1) can be rewritten as

$$
\mathbf{E}=\mathbf{E}^{w}+\mathbf{E}^{t a}+\mathbf{S}
$$

where $\mathbf{E}^{w}$ is a low-rank matrix and $\mathbf{E}^{t a}$ is a sparse matrix.

To eliminate the wall interference and form image of the target, the objective is to estimate $\mathbf{E}^{w}$ and $\mathbf{E}^{t a}$. By exploring the low-rank and sparse property of the wall clutter and target returns, the following optimization is devised

$$
\underset{\mathbf{E}^{w}, \mathbf{E}^{t a}}{\operatorname{minimize}}\left\|\mathbf{E}^{w}\right\|_{*}+\xi\left\|\mathbf{E}^{t a}\right\|_{1}+\frac{\eta}{2}\left\|\mathbf{E}-\left(\mathbf{E}^{w}+\mathbf{E}^{t a}\right)\right\|_{F}^{2},
$$

where $\xi$ is a non-negative parameter balancing the sparse constraints and the low-rank, and $\eta$ is a trade-off parameter among the low-rank, sparse components and the loss function, $\|\cdot\|_{*}$ and $\|\cdot\|_{1}$ respectively denote the nuclear norm 
and $\ell_{1}$-norm, and $\|\cdot\|_{F}$ represents the Frobenius norm. In (6), the nuclear norm is utilized to enforce the low-rank solution and $\ell_{1}$-norm is used to promote the sparse solution for the target returns.

To solve (6), the first-order conic solvers (TFOCS) [18] can be used. In [31], a Schatten $p$-norm model was proposed to deal with different rank components. To solve (6) efficiently, we propose the ADMM steps as follows.

1) An ADMM method: To solve the problem in (6), an matrix $\mathbf{A} \in\{0,1\}^{N \times T}$ is utilized, where component $\mathbf{A}(n, t)$ being 1 means that the corresponding entry is the target return, and 0 otherwise. To also efficiently handle the nuclear norm minimization (NNM), the bilinear factorization (BF) is adopted [32] and it is

$$
\operatorname{minimize}\left\|\mathbf{E}^{w}\right\|_{*}=\underset{\mathbf{U}, \mathbf{V}: \mathbf{E}^{w}=\mathbf{U V} \mathbf{V}}{\operatorname{minimize}} \frac{1}{2}\left(\|\mathbf{U}\|_{F}^{2}+\|\mathbf{V}\|_{F}^{2}\right)
$$

With (7), the problem in (6) is rewritten as

$$
\begin{aligned}
& \underset{\mathbf{U}, \mathbf{V}, \mathbf{A}, \mathbf{E}^{w}}{\operatorname{minimize}} \frac{1}{2}\|\mathbf{U}\|_{F}^{2}+\frac{1}{2}\|\mathbf{V}\|_{F}^{2}+\xi\|\mathbf{A}\|_{1} \\
&+\frac{\eta}{2}\left\|\overline{\mathbf{A}} \odot\left(\mathbf{E}-\mathbf{E}^{w}\right)\right\|_{F}^{2} \\
& \text { s.t. } \quad \mathbf{E}^{w}=\mathbf{U V}, \mathbf{A}+\overline{\mathbf{A}}=\mathbf{1} ; \mathbf{A} \text { and } \overline{\mathbf{A}} \in\{0,1\}^{N \times T},
\end{aligned}
$$

where 1 represents an all-one matrix and $\odot$ indicates element-wise multiplication operator, $\mathbf{U} \in \mathbb{R}^{N \times R}$ and $\mathbf{V} \in \mathbb{R}^{R \times T}$, and $R$ is the rank of matrix $\mathbf{E}^{w}$.

The augmented Lagrangian function of (8) is given by

$$
\begin{gathered}
\mathcal{L}\left(\mathbf{U}, \mathbf{V}, \mathbf{A}, \mathbf{E}^{w}, \boldsymbol{\lambda}\right)=\frac{1}{2}\|\mathbf{U}\|_{F}^{2}+\frac{1}{2}\|\mathbf{V}\|_{F}^{2} \\
\quad+\xi\|\mathbf{A}\|_{1}+\frac{\eta}{2}\left\|\overline{\mathbf{A}} \odot\left(\mathbf{E}-\mathbf{E}^{w}\right)\right\|_{F}^{2} \\
\quad+\frac{\beta}{2}\left\|\mathbf{E}^{w}-\mathbf{U V}\right\|_{F}^{2}+\boldsymbol{\lambda}^{T}\left(\mathbf{E}^{w}-\mathbf{U V}\right),
\end{gathered}
$$

where $\boldsymbol{\lambda}$ is the Lagrange multiplier, and $\beta$ is a penalty parameter.

By solving (9) in a recursive manner, the estimations of $\mathbf{V}, \mathbf{U}$, and $\mathbf{E}^{w}$ respectively at the $j$-th step are given 
below:

$$
\begin{aligned}
\mathbf{V}_{j+1} \leftarrow \underset{\mathbf{V}}{\operatorname{minimize}} & \frac{1}{2}\|\mathbf{V}\|_{F}^{2}+\frac{\beta_{j}}{2}\left\|\mathbf{E}_{j}^{w}-\mathbf{U}_{j} \mathbf{V}\right\|_{F}^{2} \\
& +\boldsymbol{\lambda}_{j}^{T}\left(\mathbf{E}_{j}^{w}-\mathbf{U}_{j} \mathbf{V}\right) \\
\mathbf{U}_{j+1} \leftarrow \underset{\mathbf{U}}{\operatorname{minimize}} & \frac{1}{2}\|\mathbf{U}\|_{F}^{2}+\frac{\beta_{j}}{2}\left\|\mathbf{E}_{j}^{w}-\mathbf{U V}_{j+1}\right\|_{F}^{2} \\
& +\boldsymbol{\lambda}_{j}^{T}\left(\mathbf{E}_{j}^{w}-\mathbf{U} \mathbf{V}_{j+1}\right) \\
\mathbf{E}_{j+1}^{w} \leftarrow \underset{\mathbf{E}}{\operatorname{minimize}} & \frac{\eta}{2}\left\|\mathbf{A}_{j} \odot\left(\mathbf{E}-\mathbf{E}^{w}\right)\right\|_{F}^{2} \\
& +\frac{\beta_{j}}{2}\left\|\mathbf{E}^{w}-\mathbf{U}_{j+1} \mathbf{V}_{j+1}\right\|_{F}^{2} \\
& +\boldsymbol{\lambda}_{j}^{T}\left(\mathbf{E}^{w}-\mathbf{U}_{j+1} \mathbf{V}_{j+1}\right) .
\end{aligned}
$$

It is seen that each minimization in (10) only corresponds to a single unknown parameter and Frobenius norm. Solving each corresponding optimization in (10), and after some manipulations, the solutions are

$$
\left\{\begin{array}{l}
\mathbf{V}_{j+1}=\left(\mathbf{I}+\beta_{j} \mathbf{U}_{j}^{T} \mathbf{U}_{j}\right)^{-1} \mathbf{U}_{j}^{T}\left(\beta_{j} \mathbf{E}_{j}^{w}+\boldsymbol{\lambda}_{j}\right) \\
\mathbf{U}_{j+1}=\left(\beta_{j} \mathbf{E}_{j}^{w}+\boldsymbol{\lambda}_{j}\right) \mathbf{V}_{j+1}^{T}\left(\mathbf{I}+\beta_{j} \mathbf{V}_{j+1} \mathbf{V}_{j+1}^{T}\right)^{-1} \\
\mathbf{E}_{j+1}^{w}=\frac{\eta \overline{\mathbf{A}}_{j} \odot \mathbf{E}+\beta_{j} \mathbf{U}_{j+1} \mathbf{V}_{j+1}-\boldsymbol{\lambda}_{j}}{\eta \overline{\mathbf{A}}_{j}+\beta_{j} \mathbf{1}} \\
\boldsymbol{\lambda}_{j+1}=\boldsymbol{\lambda}_{j}+\beta_{j}\left(\mathbf{E}_{j+1}^{w}-\mathbf{U}_{j+1} \mathbf{V}_{j+1}\right) \\
\beta_{j+1}=1.05 * \beta_{j} .
\end{array}\right.
$$

In (9) and (11), $\mathbf{A}$ is updated at the $j$-th step as follows:

$$
\mathbf{A}_{j+1}(n, t)=\left\{\begin{array}{l}
1, \text { if } \frac{\eta}{2}\left[\mathbf{E}-\mathbf{E}_{j}^{w}\right]_{n, t}^{2} \geq \xi \\
0, \text { otherwise. }
\end{array}\right.
$$

\section{B. Targets detection and location}

In TWR imaging, the targets' image is elliptical cloud, i.e., groups of pixels. After the clutter suppression, the target usually presents a large radar cross-section, leading to a pixels cluster with high complex amplitude values. In this work, we treat each pixels cloud as a point target. To find the positions of targets, an initialization process is introduced to locate targets from each image. It is worth noting, in this work, the number of sequential pictures in one second depends on the measurement interval $T_{s}$. For example, if the measurement interval is $T_{s}=50 \mathrm{~ms}$, based on the system settings, the image sequences will be 21 frames per second.

To efficiently perform initialization process, an observation window scheme for searching targets is developed. In a multi-frame image sequence, a global search for the first frame is required. The global search program involves to find the most energetic pixel, which is the center of a target, and the remaining targets can be recorded by 
removing the contributions of the previous targets. This process is repeated until the residual energy of the image is less than the detector threshold $T h$, given by

$$
T h=\alpha I\left(q_{\max }\right),
$$

where $\alpha$ is a constant, and $q_{\max }$ is the most energetic pixel. The output of detector is $\mathbf{d}_{k}$, given by

$$
\mathbf{d}_{k}=\left[\check{p}_{x}, \check{p}_{y}\right]^{T}
$$

where $\check{p}_{x}, \check{p}_{y}$ are the positions of the target center (the most energetic pixel) in coordinates. In doing so, a series of observation windows will be generated around the targets that will be detected after the global search. In the global search, a new window will be created to find new targets that appears in every second. The size of a window usually depends on the target's velocity. In the remaining frames, it is only necessary to check the target in each observation window sequentially. When the energy of the observation window is less than $T h$, namely the target in this window disappears, this window will be deleted. The observation windows and frame sequences are demonstrated in Fig.3.

\section{Remark:}

The size of the designed window depends on the maximum theoretical velocity $v_{\max }$ of the targets. For example, if the measurement interval $T_{s}=100 \mathrm{~ms}$, given the maximum theoretical velocity $v_{\max }=3 \mathrm{~m} / \mathrm{s}$, the maximum traveled range can be calculated to be $0.3 \mathrm{~m}$ and it can be translated into the pixel range in the image. Using that, the window size is determined.

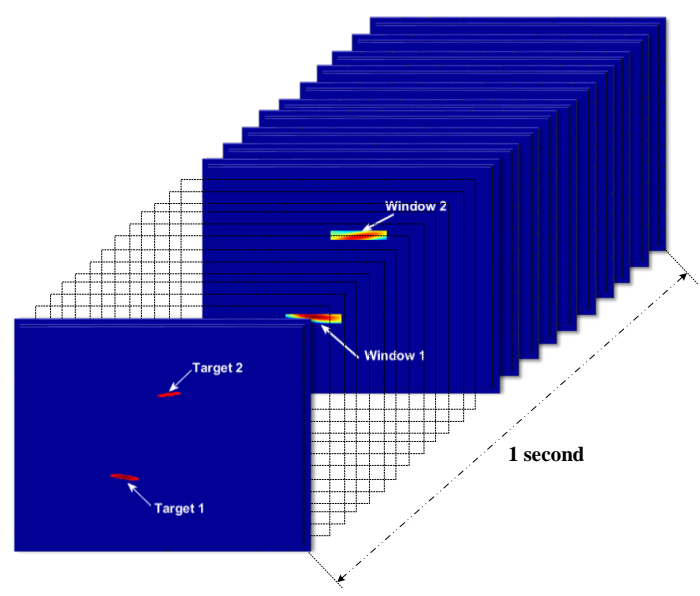

Fig. 3: TWR image frames sequence.

1) Estimation of offset: In the initialization process, the presence of the wall leads to errors in the target localization. More specifically, the speed of propagation in the wall $v$ is less than $c$, but in imaging process, full-speed (light speed) propagation is still assumed, which causes that the propagation delay $\tau_{n, q}$ to be smaller than the real one. In this case, the errors in the propagation delay $\tau_{n, q}$ lead to targets estimation errors. Wang et 
al. studied how to reduce the target offset when the wall parameters are ambiguous or unknown [33], [34]. In [1], it is discussed that the peak shift of the point spread function (PSF) is mainly on the downrange, and the offset on the crossrange is negligible. Based on this, the offset along the y-axis $p_{y}^{+}$is studied, and it can be calculated by

$$
p_{y}^{+}=(c-v) \frac{\tau_{n, w}}{2}
$$

where $v=c / \sqrt{\epsilon_{r}}$, and $\epsilon_{r}$ is the relative permittivity of wall, and $\tau_{n, w}$ is the two-way signal propagation delay between the $n$-th antenna location and the wall.

Figure 4 shows the received signal, where the propagation delay $\tau_{n, w}$ is equal to the difference between $\tau_{i n}$ and $\tau_{e x}$, which are the time points of reflection of interior wall and exterior wall. If the relative permittivity $\epsilon_{r}$ is known, $p_{y}^{+}$can be accurately calculated by (15) for homogeneous wall. With the compensation, the position vector $\mathbf{d}_{k}$ can be written as

$$
\mathbf{d}_{k}=\left[\begin{array}{c}
\check{p}_{x} \\
\check{p}_{y}
\end{array}\right]+\left[\begin{array}{c}
0 \\
p_{y}^{+}
\end{array}\right]
$$

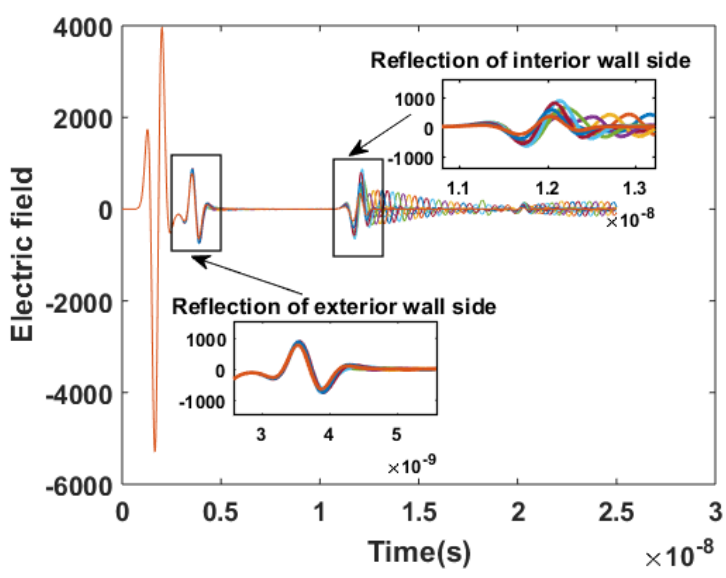

Fig. 4: Illustration of received signal.

\section{Interactive Multiple Model}

In practise, target movements is rarely governed by a single movement model. To improve the tracking accuracy, in this work, multiple models including constant velocity (CV) and constant acceleration (CA) model are utilized and the results are fused by IMM technique.

1) Constant Velocity $(\mathrm{CV})$ : In the two-dimension, target $\mathrm{x}$ can be represented by position and speed and the target state vector at time $k$ is described as

$$
\mathbf{x}_{k}=\left[p_{x}, p_{y}, \dot{p}_{x}, \dot{p}_{y}\right]^{T},
$$


where $p_{x}, p_{y}$ are the positions of the target in Cartesian coordinates, and $\dot{p}_{x}, \dot{p}_{y}$ are the speed of the target. And the target state equation is

$$
\mathbf{x}_{k+1}=\mathbf{F}_{\mathrm{CV}} \mathbf{x}_{k}+\mathbf{u}_{k}
$$

where $\mathbf{u}_{k}$ is a zero-mean white process noise sequence. The transition matrix $\mathbf{F}_{\mathrm{CV}}$ is given by

$$
\mathbf{F}_{\mathrm{CV}}=\left[\begin{array}{cccc}
1 & 0 & T_{s} & 0 \\
0 & 1 & 0 & T_{s} \\
0 & 0 & 1 & 0 \\
0 & 0 & 0 & 1
\end{array}\right],
$$

where $T_{s}$ is the sampling time.

From the initialization process, the positions of target are obtained, and hence, the measurement equation is

$$
\mathbf{d}_{k}=\mathbf{H}_{\mathrm{CV}} \mathbf{x}_{k}+\mathbf{n}_{k}
$$

where $\mathbf{n}_{k}$ is the measurement noise and

$$
\mathbf{H}_{\mathrm{CV}}=\left[\begin{array}{llll}
1 & 0 & 0 & 0 \\
0 & 1 & 0 & 0
\end{array}\right] .
$$

2) Constant Acceleration (CA): The CA model is introduced to deal with the accelerations in the movement, the target state vector $\mathbf{x}$ at time $k$ is defined as

$$
\mathbf{x}_{k}=\left[p_{x}, p_{y}, \dot{p}_{x}, \dot{p}_{y}, \ddot{p}_{x}, \ddot{p}_{y}\right]^{T}
$$

where $\ddot{p}_{x}, \ddot{p}_{y}$ denote the accelerations along the $x$ and $y$, respectively. Similarly, the transition function for the CA model is

$$
\mathbf{x}_{k+1}=\mathbf{F}_{\mathrm{CA}} \mathbf{x}_{k}+\mathbf{u}_{k}
$$

where the transition matrix $\mathbf{F}_{\mathrm{CA}}$ is described as

$$
\mathbf{F}_{\mathrm{CA}}=\left[\begin{array}{cccccc}
1 & 0 & T_{s} & 0 & T_{s}^{2} / 2 & 0 \\
0 & 1 & 0 & T_{s} & 0 & T_{s}^{2} / 2 \\
0 & 0 & 1 & 0 & T_{s} & 0 \\
0 & 0 & 0 & 1 & 0 & T_{s} \\
0 & 0 & 0 & 0 & 1 & 0 \\
0 & 0 & 0 & 0 & 0 & 1
\end{array}\right] .
$$


The linear observation matrix $\mathbf{H}_{\mathrm{CA}}$ for CA model is given as follow:

$$
\mathbf{H}_{\mathrm{CA}}=\left[\begin{array}{cccccc}
1 & 0 & 0 & 0 & 0 & 0 \\
0 & 1 & 0 & 0 & 0 & 0
\end{array}\right] .
$$

3) Interactive Multiple Model (IMM): In the IMM method, the combination of state estimations from each filters under different models is implemented by a Markovian model. From Figure 5, the IMM method contains four steps of input model interaction, filtering, model probability update, and state estimations combination, and each step is provided as follows.

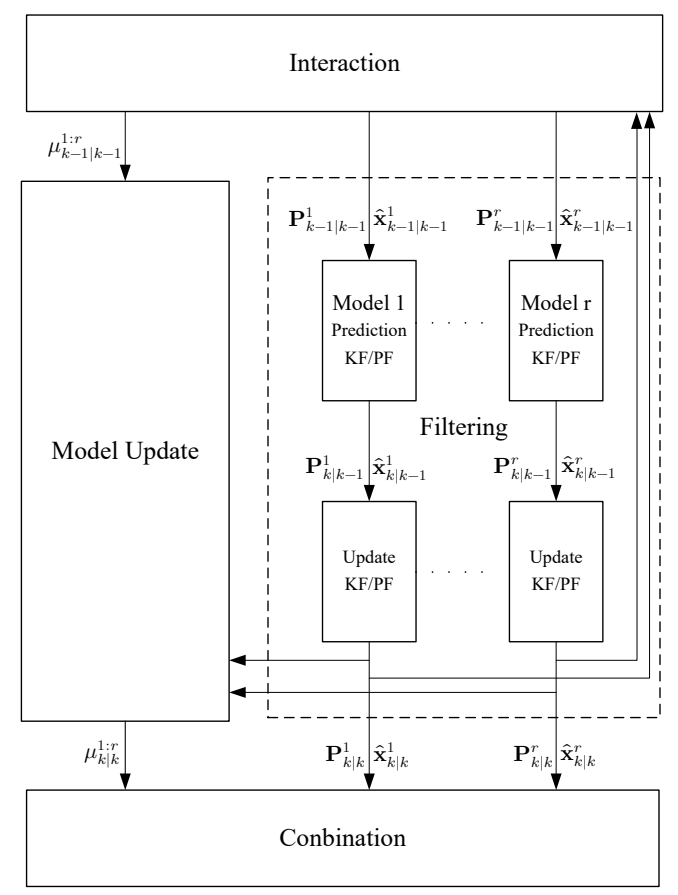

Fig. 5: System diagram of IMM-based filters.

- Interaction: Assume that $r$ motion models are involved in the IMM algorithm. The mixing probabilities $\mu_{k-1 \mid k-1}^{j \mid i}$ for model $i$ and model $j$ can be written as:

$$
\mu_{k-1 \mid k-1}^{j \mid i}=\frac{1}{\bar{c}_{j}} p_{i j} \mu_{k-1}^{i}
$$

where $\mu_{k-1}^{i}$ is the probability of model $i$, and $p_{i j}$ represents the transition probability from model $i$ to model $j$, and $\bar{c}_{j}$ is a normalization factor, given by

$$
\bar{c}_{j}=\sum_{i=1}^{r} p_{i j} \mu_{k-1}^{i}
$$


With that, the initial inputs for each model at time $k$ are

$$
\begin{aligned}
& \overline{\mathbf{x}}_{k-1 \mid k-1}^{i}=\sum_{j} \mu_{k-1 \mid k-1}^{j \mid i} \hat{\mathbf{x}}_{k-1 \mid k-1}^{j} \\
& \overline{\mathbf{P}}_{k-1 \mid k-1}^{i}=\sum_{j} \mu_{k-1 \mid k-1}^{j \mid i}\left\{\mathbf{P}_{k-1 \mid k-1}^{j}+\right. \\
& \left.\left(\overline{\mathbf{x}}_{k-1 \mid k-1}^{i}-\hat{\mathbf{x}}_{k-1 \mid k-1}^{j}\right) \times\left(\overline{\mathbf{x}}_{k-1 \mid k-1}^{i}-\hat{\mathbf{x}}_{k-1 \mid k-1}^{j}\right)^{T}\right\},
\end{aligned}
$$

where $\hat{\mathbf{x}}_{k-1 \mid k-1}^{j}, \mathbf{P}_{k-1 \mid k-1}^{j}$ are the state estimate and covariance of model $j$ at time $k-1$, respectively.

- Filtering: According to the initial inputs in interaction step, the new estimates of $\hat{\mathbf{x}}_{k \mid k}^{i}$ and $\mathbf{P}_{k \mid k}^{i}$ are calculated using the KF/PF, which are described below.

- Model Probability Update: For model $i$, the maximum likelihood function $\Lambda_{k}^{i}=\mathbb{N}\left(\mathbf{v}_{k}^{i} ; \mathbf{0}, \mathbf{S}_{k}^{i}\right)$ is adopted to update the probability of each model, where $\mathbb{N}(\cdot)$ is Gaussian distribution, $\mathbf{v}_{k}^{i}$ and $\mathbf{S}_{k}^{i}$ are the measurement residual and its covariance, respectively. The probability $\mu_{k \mid k}^{i}$ at time $k$ is updated

$$
\begin{aligned}
\mu_{k \mid k}^{i} & =\frac{1}{c} \Lambda_{k}^{i} \bar{c}_{i} \\
c & =\sum_{i} \Lambda_{k}^{i} \bar{c}_{i} .
\end{aligned}
$$

- Combination: The combined state estimates of $\hat{\mathbf{x}}_{k \mid k}$ and $\mathbf{P}_{k \mid k}$ from different models are produced by

$$
\begin{aligned}
\hat{\mathbf{x}}_{k \mid k} & =\sum_{i} \mu_{k \mid k}^{i} \hat{\mathbf{x}}_{k \mid k}^{i} \\
\mathbf{P}_{k \mid k} & =\sum_{i} \mu_{k \mid k}^{i}\left\{\mathbf{P}_{k \mid k}^{i}+\left(\hat{\mathbf{x}}_{k \mid k}-\hat{\mathbf{x}}_{k-1 \mid k-1}^{i}\right)\right. \\
& \left.\times\left(\hat{\mathbf{x}}_{k \mid k}-\hat{\mathbf{x}}_{k \mid k}^{i}\right)^{T}\right\}
\end{aligned}
$$

\section{Target tracking}

1) $K F$ : The KF is a widely used filter due to its simplicity and effectiveness. The KF process is recursively performed using the prediction from the transition equation and update from the measurement equation [35].

Suppose the state $\hat{\mathbf{x}}_{k-1 \mid k-1}$ at time $k-1$ is estimated, by a use of transition matrix, the predicted state $\hat{\mathbf{x}}_{k \mid k-1}$ is

$$
\begin{aligned}
& \hat{\mathbf{x}}_{k \mid k-1}=\mathbf{F} \hat{\mathbf{x}}_{k-1 \mid k-1}, \\
& \mathbf{P}_{k \mid k-1}=\mathbf{Q}_{k-1}+\mathbf{F} \mathbf{P}_{k-1 \mid k-1} \mathbf{F}^{T},
\end{aligned}
$$

where $\mathbf{P}_{k-1 \mid k-1}$ and $\mathbf{P}_{k \mid k-1}$ respectively indicate the error covariance matrix and the predicted error covariance matrix.

When the new measurement $\mathbf{d}_{k}$ at time $k$ is available, the update step conducts as 


$$
\begin{aligned}
& \hat{\mathbf{x}}_{k \mid k}=\hat{\mathbf{x}}_{k \mid k-1}+\mathbf{K}_{k}\left(\mathbf{d}_{k}-\mathbf{H} \hat{\mathbf{x}}_{k \mid k-1}\right) \\
& \mathbf{P}_{k \mid k}=\mathbf{P}_{k \mid k-1}-\mathbf{K}_{k} \mathbf{H P}_{k \mid k-1} \\
& \mathbf{K}_{k}=\mathbf{P}_{k \mid k-1} \mathbf{H}^{T}\left(\mathbf{H}_{k} \mathbf{P}_{k \mid k-1} \mathbf{H}^{T}+\mathbf{R}_{k}\right)^{-1},
\end{aligned}
$$

where $\mathbf{K}_{k}$ is called the Kalman gain.

2) $P F:$ PF is known to handle the nonlinear and non-Gaussian property in the systems and also provides a better tracking accuracy than the KF. Even though the state/measuremt models used in (18) and (20) are linear, it is still explored in this work to demonstrate its potential. The objective of tracking is to obtain the posterior probability distribution function (PDF) of the state, i.e., $\pi\left(\mathbf{x}_{k} \mid \mathbf{d}_{1: k}\right)$, where $\mathbf{d}_{1: k}=\left\{\mathbf{d}_{1}, \mathbf{d}_{2}, \cdots, \mathbf{d}_{k}\right\}$ represents the observations up to the time $k$. The initial density of the state vector is denoted by $\pi\left(\mathbf{x}_{0}\right)=\pi\left(\mathbf{x}_{0} \mid \mathbf{d}_{0}\right)$, where $\mathbf{d}_{0}$ indicates no measurements. The PDF $\pi\left(\mathbf{x}_{k} \mid \mathbf{d}_{1: k}\right)$ is also calculated recursively in two steps of prediction and update. Assume the PDF $\pi\left(\mathbf{x}_{k-1} \mid \mathbf{d}_{1: k-1}\right)$ at time $(k-1)$ is estimated, the predicted density of the state at time $k$ is computed by

$$
\pi\left(\mathbf{x}_{k} \mid \mathbf{d}_{1: k-1}\right)=\int p\left(\mathbf{x}_{k} \mid \mathbf{x}_{k-1}\right) \pi\left(\mathbf{x}_{k-1} \mid \mathbf{d}_{1: k-1}\right) d \mathbf{x}_{k-1}
$$

where $p\left(\mathbf{x}_{k} \mid \mathbf{x}_{k-1}\right)$ is the transition probability function.

At time $k$, when the observation $\mathbf{d}_{k}$ is available, via the Bayes' rule, an update is performed as

$$
\pi\left(\mathbf{x}_{k} \mid \mathbf{d}_{1: k}\right) \propto p\left(\mathbf{d}_{k} \mid \mathbf{x}_{k}\right) \pi\left(\mathbf{x}_{k} \mid \mathbf{d}_{1: k-1}\right)
$$

In (33), the integration is usually multi-dimensional, which means that the closed-form solution is difficult to obtain in general. The PF applies a set of random samples with associated weights and computes estimates using these samples and weights. With a large set of $N_{p}$ particles $\left\{\mathbf{x}_{k-1}^{(i)}\right\}_{i=1}^{N_{p}}$ and their associated weights $\left\{w_{k-1}^{(i)}\right\}_{i=1}^{N_{p}}$, the posterior density at time $k-1$ is approximated as

$$
\pi\left(\mathbf{x}_{k-1} \mid \mathbf{d}_{1: k-1}\right) \approx \sum_{i=1}^{N_{p}} w_{k-1}^{(i)} \delta\left(\mathbf{x}_{k-1}-\mathbf{x}_{k-1}^{(i)}\right)
$$

where $\delta(\cdot)$ is the Dirac delta function.

Using a properly designed proposal function $q(\cdot)$, the new particles $\left\{\mathbf{x}_{k}^{(i)}\right\}_{i=1}^{N_{p}}$ are generated

$$
\mathbf{x}_{k}^{(i)} \sim q\left(\mathbf{x}_{k} \mid \mathbf{x}_{k-1}^{(i)}, \mathbf{d}_{1: k}\right), \quad i=1, \cdots, N_{p}
$$

With the new particles, the importance weight $w_{k}^{(i)}$ is calculated by

$$
w_{k}^{(i)} \propto w_{k-1}^{(i)} \times \frac{p\left(\mathbf{d}_{k} \mid \mathbf{x}_{k}^{(i)}\right) p\left(\mathbf{x}_{k}^{(i)} \mid \mathbf{x}_{k-1}^{(i)}\right)}{q\left(\mathbf{x}_{k} \mid \mathbf{x}_{k-1}^{(i)}, \mathbf{d}_{1: k}\right)} .
$$


From the new particles and the newly computed weights, the state estimate at time $k$ using minimum mean square error (MMSE) criterion is [36]

$$
\hat{\mathbf{x}}_{k}=\mathbb{E}\left[\mathbf{x}_{k} \mid \mathbf{d}_{1: k}\right]=\int \mathbf{x}_{k} \pi\left(\mathbf{x}_{k} \mid \mathbf{d}_{1: k}\right) d \mathbf{x}_{k} \approx \sum_{i=1}^{N} w_{k}^{(i)} \mathbf{x}_{k}^{(i)},
$$

where $\mathbb{E}$ denotes the expectation operator. If the transition function is used as the proposal function, the weight update simply becomes $w_{k}^{(i)} \propto w_{k-1}^{(i)} \times p\left(\mathbf{d}_{k} \mid \mathbf{x}_{k}^{(i)}\right)$.

\section{Remarks:}

1. Every target is independently assigned a KF and a PF tracker, and their performances are evaluated when tracking processes are terminated.

2. To reduce the search computation, each observation window corresponds to a tracking process, and an initiation of a tracking process will only occur at the first frame of every second. In other word, the birth of tracking process occurs at the first frame of every second.

3. A tracking process is terminated if one of the following conditions is satisfied:

1) observation window is deleted;

2) the velocity of target has reached the velocity threshold;

3) the target trajectory remains still for $K$ frames.

\section{EXPERIMENTAL RESULTS}

In this section, the experimental results, including clutter reduction, image formation and target tracking, are provided to verify the performance with different scenarios. In the experiments, the radar dataset is obtained using the gprMax [37].

\section{A. The effect of clutter reduction}

In this section, the wall effects including the wall material and wall thickness are examined. The geometry setup for evaluating the effect of wall parameters for clutter reduction is shown in Fig. 6, where the transceiver antenna is positioned at $0.07 \mathrm{~m}$ in downrange. The front-wall is $3 \mathrm{~cm}$ away from the antenna, and a cylinder target with a radius of $6.25 \mathrm{~cm}$ resembling a human is placed at $(1.5,1.045)$, i.e., $M=1$. In order to simulate the EM reflection effect of muscles, the relative permittivity of target is 96 [38]. The imaged scene from $[0.07,2] \mathrm{m}$ in downrange and $[0,3] \mathrm{m}$ in crossrange is considered.

During the scan, the receiver antenna at 101 positions are uniformly collected from $0.3 \mathrm{~m}$ to $2.8 \mathrm{~m}$ along the crossrange. The TWR radiation signal is a Ricker waveform with its center frequency being $1 \mathrm{GHz}$. With the cross-validation, our proposed method is found to consistently provide reasonable results, when $\eta \in[5,20]$ and 
$\xi \in[0.1,1]$. Therefore, in the following experiments, the parameters of $\eta=10, \xi=1, r=5$, and the initial value of $\beta=1$ are selected. To subjectively evaluate the performance, the image quality using the target-to-clutter ratio

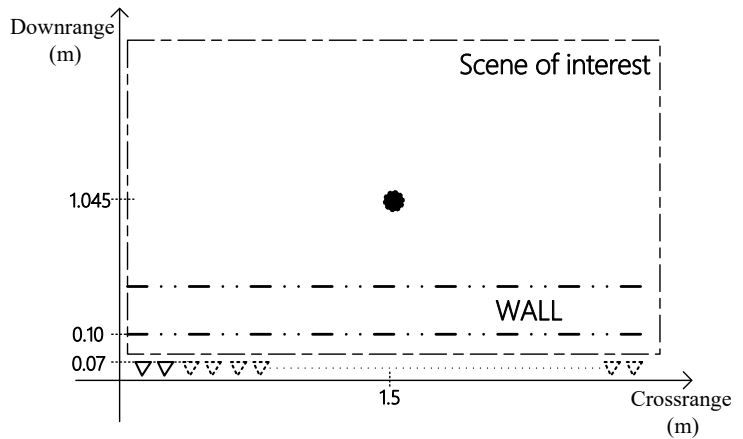

Fig. 6: Geometry for experimental setup

(in $\mathrm{dB}$ ) is defined as

$$
\mathrm{TCR}=10 \log _{10}\left(\frac{\frac{1}{N_{t}} \sum_{q \in R_{t}}|I(q)|^{2}}{\frac{1}{N_{c}} \sum_{q \in R_{c}}|I(q)|^{2}}\right),
$$

where $R_{t}$ indicates the target region, $R_{c}$ represents the entire image excluding the target region, and $N_{t}$ and $N_{c}$ respectively are the numbers of pixels in the target and clutter regions.

In the first experiment, the effect of relative permittivity $\epsilon_{r}$ is studied, by fixing the thickness of homogeneous wall to be $10 \mathrm{~cm}$. Two different kinds of wooden walls are utilized, one grit wall and two concrete walls with different moisture contents. The results are obtained by averaging over 10 independent runs and summarized in Table I. It is noticed that TCRs are insensitive to the parameter of the relative permittivity. In Table II, the TCR values are provided in terms of different thickness, but with a fixed permittivity $\epsilon_{r}=6$. As expected, the TCR decreases when the thickness increases. This is to say that the thickness plays a lager role than the permittivity, where the reflected signal is attenuated more as the thickness increases. Finally, Table III lists the experimental

TABLE I: TCR with different relative permittivity

\begin{tabular}{c|c}
\hline Wall material & TCR $(\mathbf{d B})$ \\
\hline Wood with $\epsilon_{r}=2.1$ & 17.301 \\
\hline Wood with $\epsilon_{r}=3.4$ & 17.241 \\
\hline Grit with $\epsilon_{r}=3.7$ & 17.217 \\
\hline Concrete with $\epsilon_{r}=5.3$ & 17.043 \\
\hline Concrete with $\epsilon_{r}=6$ & 16.981 \\
\hline
\end{tabular}

results of different wall structures. The hollow bricks are often utilized for building interior walls and reinforced concrete is used for exterior walls. The sandwich structure means that the surface and interior of the wall are two different materials, and the double layer structure indicates a wall of two materials. Based on these structures, the TCRs are now computed and it is seen that the effect of wall structures is also not significant. 
TABLE II: TCR with different thickness

\begin{tabular}{c|c}
\hline Thickness of wall (m) & TCR (dB) \\
\hline 0.10 & 16.981 \\
\hline 0.15 & 16.606 \\
\hline 0.20 & 15.833 \\
\hline 0.30 & 15.594 \\
\hline 0.40 & 12.751 \\
\hline 0.60 & 8.451 \\
\hline
\end{tabular}

TABLE III: TCR for different wall structures

\begin{tabular}{c|c}
\hline Type of wall & TCR (dB) \\
\hline Hollow brick & 16.359 \\
\hline Reinforced concrete & 16.513 \\
\hline Sandwich wall & 15.416 \\
\hline Double layer & 16.672 \\
\hline
\end{tabular}

It is concluded from the conducted experiments, the effects of materials and structures of the wall are negligible, and the thickness of the wall is a more deciding factor.

Additionally, the performance of our proposed method under different signal-to-noise ratios (SNRs) is studied. In the spirit of making the clutter reduction more challenging, $20 \%$ or $40 \%$ data are randomly discarded to simulate the missing elements and only 51 antenna positions are utilized. The results are averaged over 100 independent trials and provided in Fig.7. It is seen that TCR is of good performance when SNR is larger than 10dB. In a more harsh case, with only 51 antenna are available and $40 \%$ missing measurements, TCR still larger than 10dB with 0dB SNR. This result shows the robustness of the proposed method against the noise and data missing. Meanwhile, to further demonstrate the capability of the proposed method, the images obtained by the proposed method under different SNRs are depicted in Fig.8, with 51 antenna positions and 40\% missing. Even under the low SNR condition, say $0 \mathrm{~dB}$, the proposed method is still able to produce the peak at the target position.

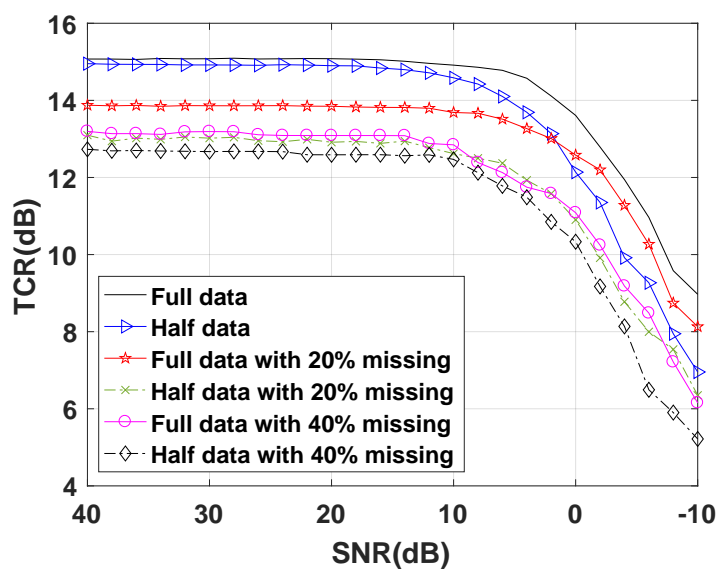

Fig. 7: TCR versus SNR. 


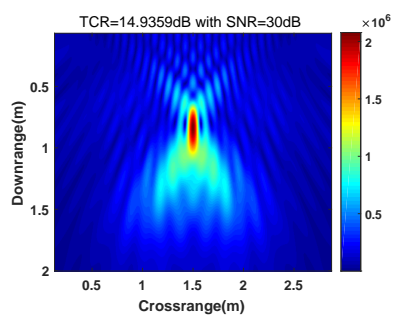

(a)

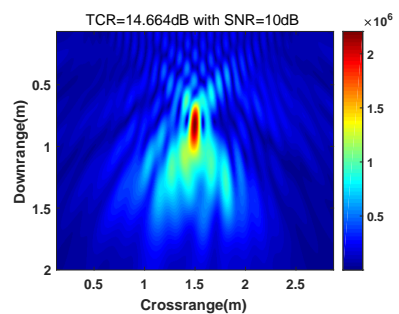

(c)

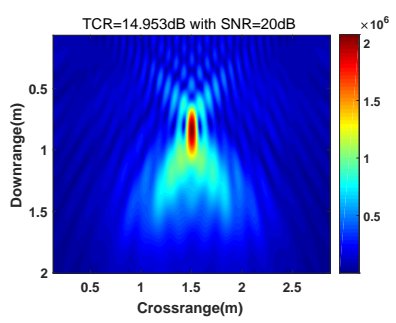

(b)

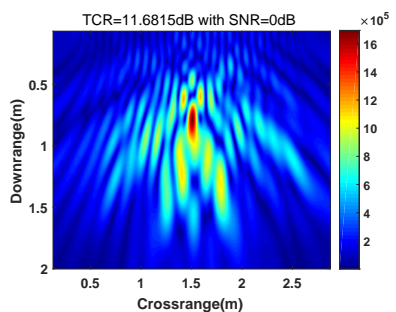

(d)

Fig. 8: The images are formed by 51 antenna positions with $40 \%$ data missing. (a) SNR=30dB, (b) SNR=20dB, (c) $\mathrm{SNR}=10 \mathrm{~dB}$, (d) $\mathrm{SNR}=0 \mathrm{~dB}$.

\section{B. The result of TWR imaging}

In this experiment, the TWR system is mounted on a vehicle, a $10 \mathrm{~cm}$ thickness hollow brick wall with $\epsilon_{r}=6$ is positioned at $1.5 \mathrm{~m}$ in downrange, which is $1.4 \mathrm{~m}$ away from the transceiver antenna. A cylinder target of $6.25 \mathrm{~cm}$ radius is positioned at $(1.3,1.845)$, i.e., $M=1$. After the clutter reduction, the final formed images of different approaches are provided in Fig. 9. It can clearly be observed that a strong point is detected in the scene, demonstrated in Fig. 9(a). For a comparison purpose, the results of iterative soft thresholding method [10], background subtraction [1] and SVD method [7] are depicted in Fig. 9(b) to (d). In Fig. 9(b), the image obtained by approach in [10] that is based on low-rank and sparse model has a slight tailing. Although background subtraction yields a higher TCR, its performance heavily relies on the availability of the empty scene, which is not possible in real applications. In Fig. 9(d), the result obtained by SVD method [7] is provided, in which several ghost targets appear.

In the second experiment, the results of two targets are presented in Fig. 10, where a $4.2 \mathrm{~cm}$ cube target at $(1.8,2.1)$ and a cylinder target of $3.15 \mathrm{~cm}$ is at $(0.9,1.885)$ are used. The images obtained by different approaches are depicted in Figure 10(a) to (d). It is observed that the strong wall reflections are removed by each method. One can notice that our method produces slightly lower TCR than that of background subtraction method. On the other hand, our method clearly detected two targets, and the iterative soft thresholding approach [10] and SVD method [7] both missed the second target, shown in Fig. 10(b) and (d). 


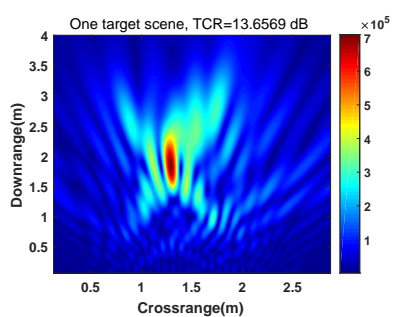

(a)

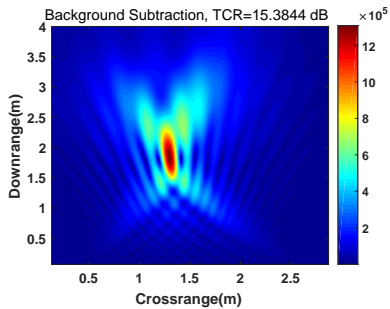

(c)

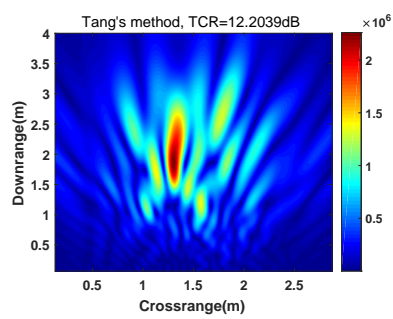

(b)

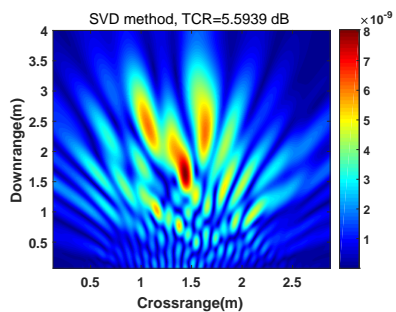

(d)

Fig. 9: The results of the one target scene: (a) image by the proposed method, (b) image by Tang's method [10], (c) image by background subtraction [1], (d) image by SVD method [7].

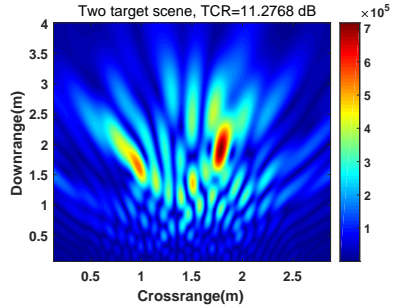

(a)

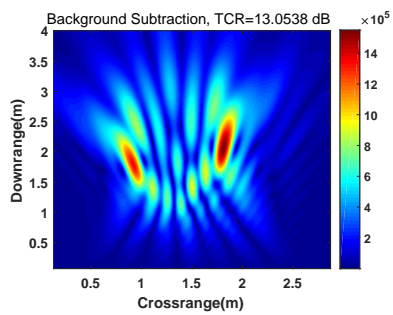

(c)

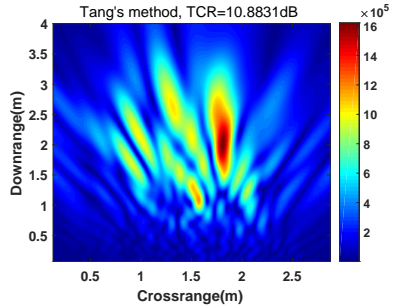

(b)

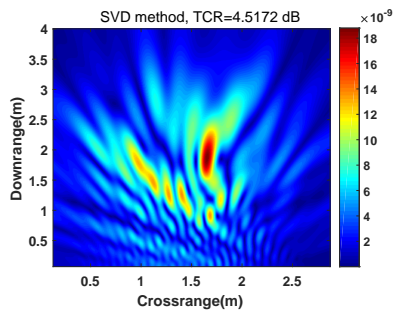

(d)

Fig. 10: The results of the two targets scene: (a) image by the proposed method, (b) image by Tang's method [10], (c) image by background subtraction [1], (d) image by SVD method [7].

\section{The result of TWR tracking}

In this experiment, we consider the tracking scenario of indoor targets with constant velocity (CV) and constant acceleration (CA) movements. The size of the room are $l_{x}=2 \mathrm{~m}$ and $l_{y}=1.5 \mathrm{~m}$ that is surrounded by $30 \mathrm{~cm}$-thick concrete walls with $\epsilon_{r}=6$. The TWR antenna is uniformly collected from $0.3 \mathrm{~m}$ to $2.8 \mathrm{~m}$ along the crossrange. In one frame, the target is assumed to be stationary, but it moves between frames. Therefore, the Doppler effect will not cause image blur in our case. The remaining parameters in the experiments are provided in Table IV. 
TABLE IV: Parameters setting

\begin{tabular}{ccc}
\hline Parameter & Value & Specification \\
\hline$T_{s}$ & $50 \mathrm{~ms}$ & Sampling time \\
$\alpha$ & 0.75 & Threshold rate \\
$f_{c}$ & $1 \mathrm{GHz}$ & Center frequency \\
$t_{f}$ & $30 \mathrm{~ns}$ & Fast-time of TWR measurement \\
$l_{o w}$ & $0.4 \mathrm{~m}$ & Length of observation window \\
$r_{t}$ & $0.03 \mathrm{~m}$ & Radius of target \\
$\epsilon_{t a}$ & 96 & Relative permittivity of target \\
$N_{p}$ & 10000 & Particle number of PF \\
\hline
\end{tabular}

In the IMM, we consider $r=2$ models, $\mathrm{CV}$ and $\mathrm{CA}$, for tracking the target, and the transition probability matrix between these models is

$$
\mathbf{P}=\left[\begin{array}{ll}
0.97 & 0.03 \\
0.03 & 0.97
\end{array}\right]
$$

For the tracking purpose, the 4 by 4 noise covariance matrix $\mathbf{Q}_{\mathrm{CV}}$ for $\mathrm{CV}$ model, the 6 by 6 noise covariance matrix $\mathbf{Q}_{\mathrm{CA}}$ for $\mathrm{CA}$ model, and 2 by 2 matrix $\mathbf{R}$ in $\mathrm{KF}$ are given below:

$$
\begin{gathered}
\mathbf{Q}_{\mathrm{CV}}=\operatorname{diag}\{0.5,0.5,1.5,1.5\} \\
\mathbf{Q}_{\mathrm{CA}}=\operatorname{diag}\{0.5,0.5,0.5,0.5,1.5,1.5\}, \\
\mathbf{R}=\left[\begin{array}{cc}
0.001 & 0 \\
0 & 0.7
\end{array}\right] .
\end{gathered}
$$

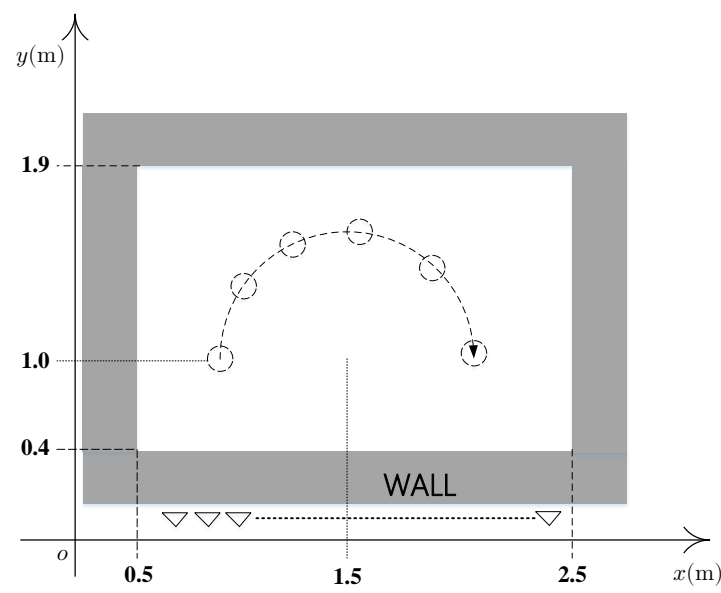

Fig. 11: Single target scenario: The dashed circles depict the target positions.

In Fig.11, the single target tracking scenario is studied first, where target moves from $(1.0,1.0) \mathrm{m}$ to $(2.0,1.0) \mathrm{m}$ to form a semicircular trajectory with the center at $(1.5,1.0) \mathrm{m}$, and the traveling velocity is $v_{t}=1.5 \mathrm{~m} / \mathrm{s}$. Based on this setup, it is calculated that target moves 2 seconds long. To study the influence of the number of antenna elements on the tracking accuracy, we used $N=26,51,76,101,126,151$ antenna positions in this scene to conduct 
the tracking task, and the results are provided in Fig. 12. It is noticed that the accuracy improves as the number of antennas increases. To balance the efficiency and accuracy of tracking, in the following experiment, 51 antenna positions are used. To visually see the image, six frames of TWR images in this snapshot sequence with sequence number of $k=1,8,16,20,32,41$ are provided in Fig.13. It is seen that the proposed target tegion detector captures the target movements.

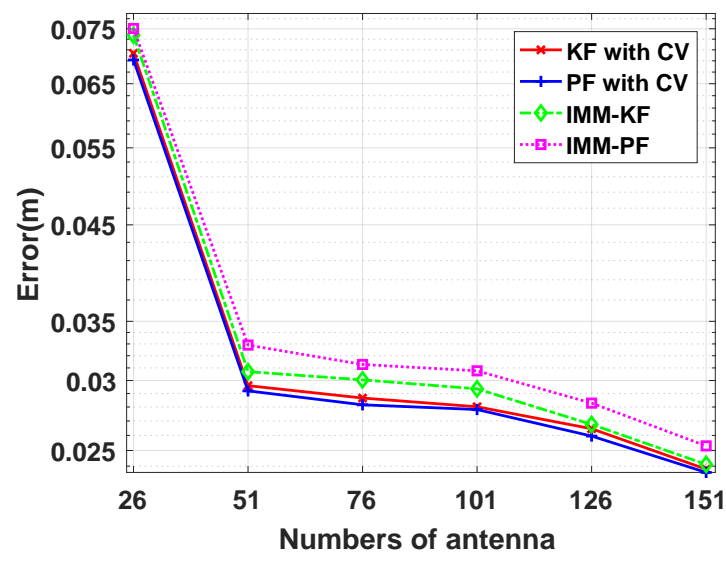

Fig. 12: The effect of the number of antennas on tracking accuracy.

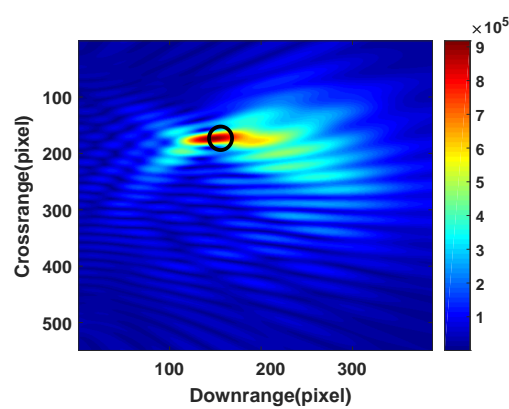

(a)

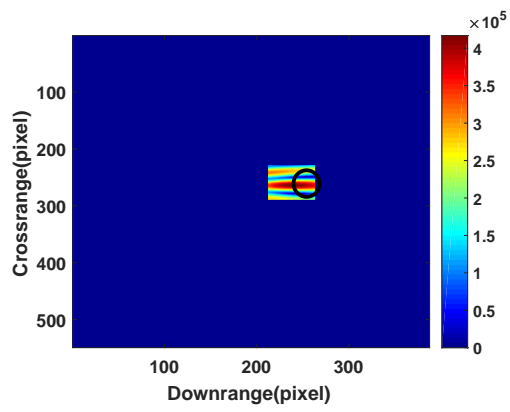

(d)

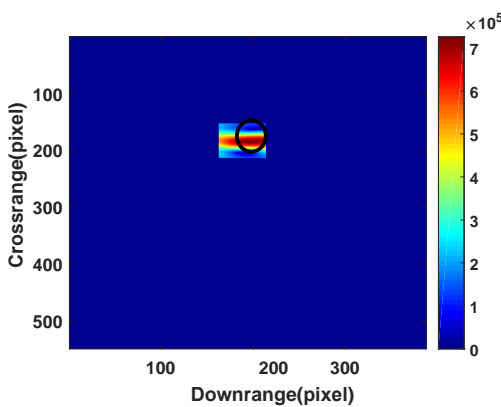

(b)

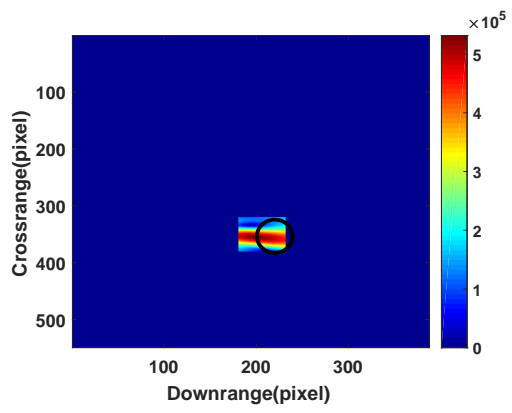

(e)

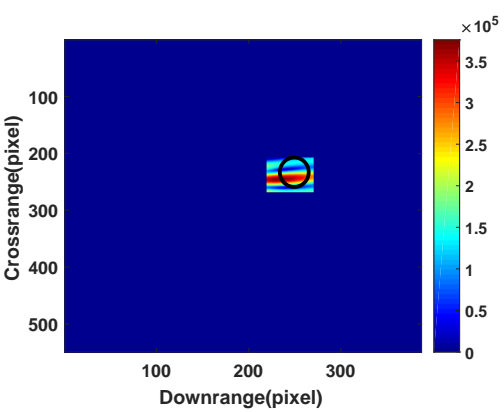

(c)

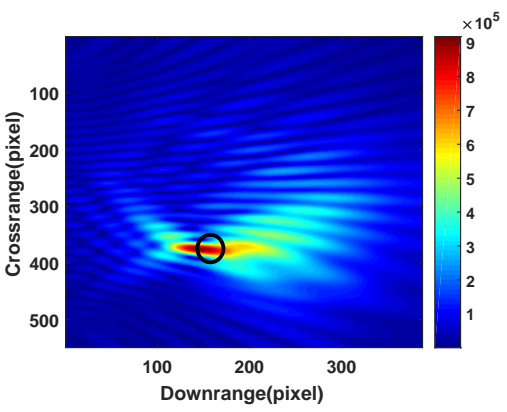

(f)

Fig. 13: TWR images of sequence in the single target scenario. The solid circle depicts the true position. (a) $k=1$, (b) $k=8$, (c) $k=16$, (d) $k=20$, (e) $k=32$, (f) $k=41$.

At the same time, it is also seen that the pixel cloud of the corresponding target always appears on the left side of the solid circle. It means that the detected target position is slightly deviated in the downrange, which is result 
by the propagation delay through the front wall. To compensate this offset, using (15), the location error caused by the front wall is estimated. Finally, the compensated measurement data are utilized to perform the tracking task by a use of the IMM-KF, IMM-PF, and also KF and PF with the single CV model. The tracking results are now depicted in Fig.14. The corresponding distance errors for each filter are provided in Figure 15.
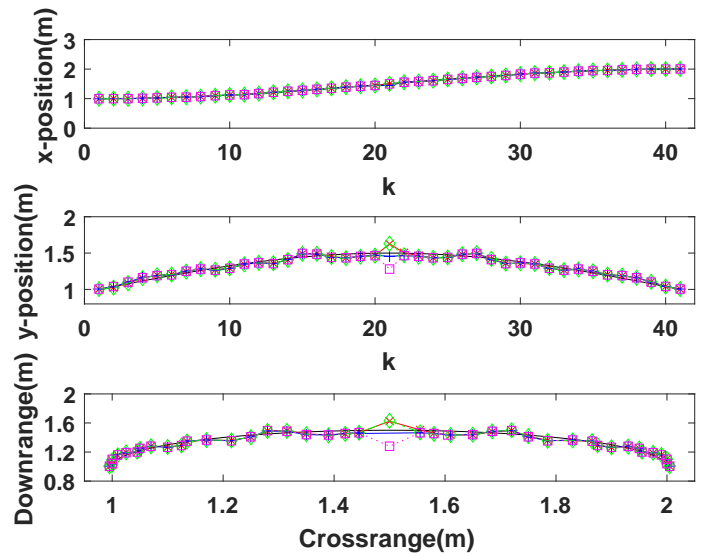

Fig. 14: Outputs of the tracker and the ground truth in the single target scenario. The top row indicates the target position along $x$. The middle row indicates the target position along $y$. The bottom row indicates the real trajectory. '-': real trajectory; +: PF with $\mathrm{CV}$; $\times$ : KF with $\mathrm{CV}$; $\square$ : IMM-PF; $\diamond:$ IMM-KF.

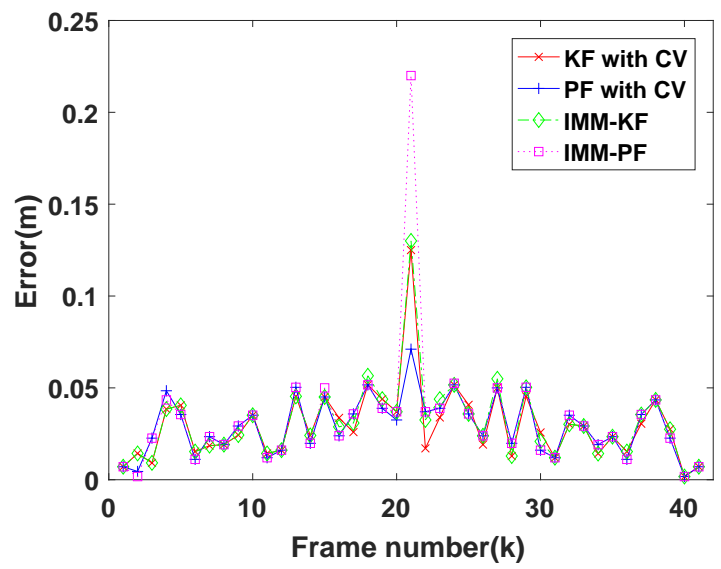

Fig. 15: Distance errors for each filter under CV model.

It is noticed that the outputs of trackers along $x$ (crossrange) is nearly perfect and it matches the true positions well. The PF with CV model presents a small offset before and after frame 10, which corresponds to the farthest position of the target from the antenna. In comparison, there are slightly offset between the outputs of the trackers and ground truth along $y$ (downrange). This is because of the TWR bandwidth limitation and TWR imaging setup that faces the antenna. However, this tracking error is acceptable given the current TWR system [39]. It is seen that the $\mathrm{KF} / \mathrm{PF}$ with single $\mathrm{CV}$-model has more better performance than IMM based filters, which is consistent with 
the true scenario because the movements follow the CV model, demonstrated in Figure 15. The average tracking accuracy for each model are given in Table $\mathrm{V}$ and it is seen that the accuracy is in the range of a few centimeters.

In the second experiment, the movement obeys a random walk. At the constant speed $v_{t}=1.5 \mathrm{~m} / \mathrm{s}$, we randomly generate the direction of the target at each moment. The tracking results within two seconds are demonstrated in Fig.16. It can be seen that each tracker maintains a good performance in this task, which indicates that the tracking trajectory is very close to the real one. Moreover, in this case, the PF produces a better tracking performance, meaning that tracking error is smaller, also indicated in Table V. The Figure 17 shows the estimation errors for each filter, where they all produce a comparable performance.

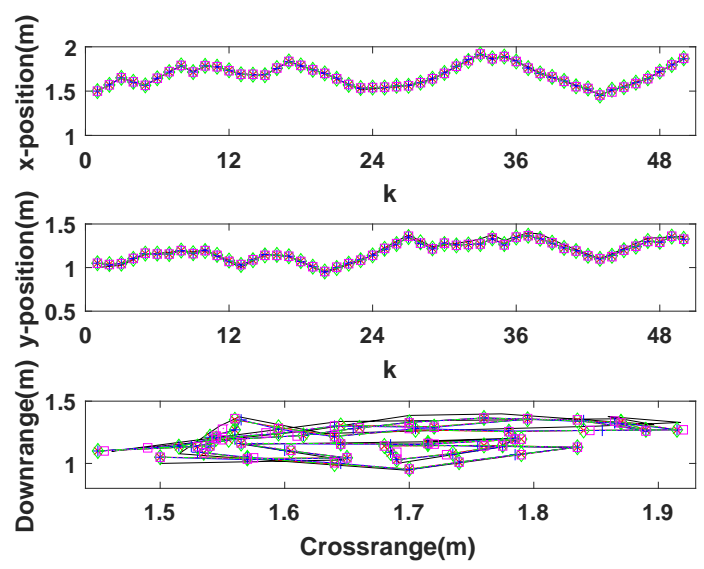

Fig. 16: Outputs of the tracker and the ground truth in the random walk. The top row indicates the target position along $x$. The middle row indicates the target position along $y$. The bottom row indicates the real trajectory. '-': real trajectory; + : PF with $\mathrm{CV} ; \times$ : KF with $\mathrm{CV}$; $\square$ : IMM-PF; $\diamond:$ IMM-KF.

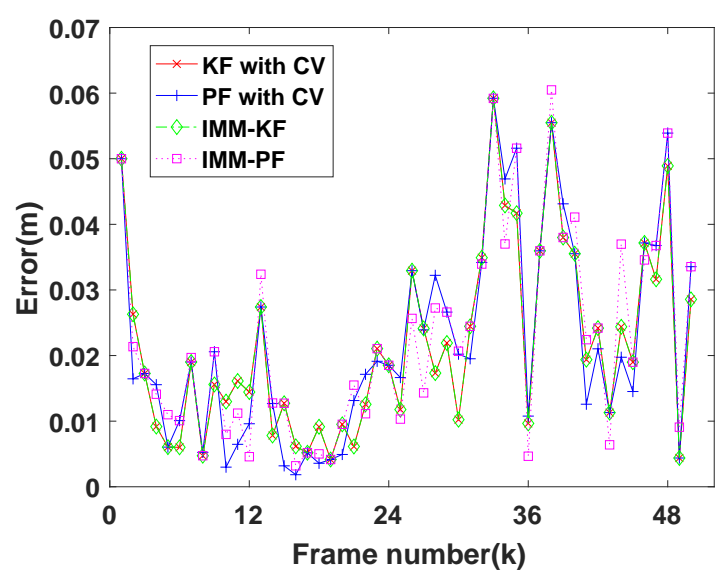

Fig. 17: Distance errors for each filter under the random walk.

Next, the CV-CA-CV movement model is considered. The target is assumed to travel at a constant speed $v_{t}=$ $0.5 \mathrm{~m} / \mathrm{s}$ in the first second, and follow a CA movement in the next 0.6 second, where the target accelerates in the first 0.4 second at the acceleration $a=0.2 \mathrm{~m} / \mathrm{s}^{2}$ and then decelerates at $a=-0.3 \mathrm{~m} / \mathrm{s}^{2}$. Finally, the the 
target returns back to the CV movement. The tracking results are plotted in Fig.18. It is obvious, in this case, that IMM-KF/IMM-PF produce the better tracking performance. Judging from estimation error in Figure 19, the same conclusion can be drawn as well that IMM based model presents the best tracking ability thanks to the model fitness.

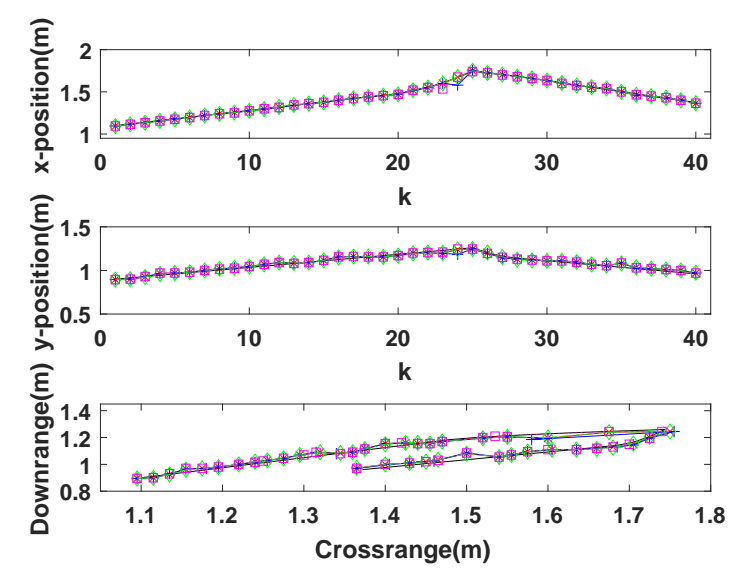

Fig. 18: Outputs of the tracker and the ground truth in the CV-CA-CV movement model. The top row indicates the target position along $x$. The middle row indicates the target position along $y$. The bottom row indicates the real trajectory. '-': real trajectory; +: PF with CV; $\times$ : KF with CV; $\square$ : IMM-PF; $\diamond:$ IMM-KF.

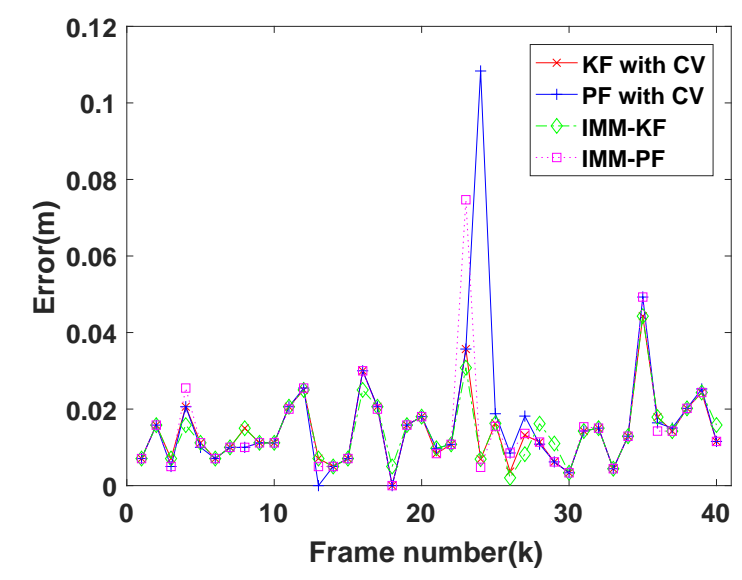

Fig. 19: Distance errors for each filter under the CV-CA-CV movement.

To further demonstrate the capacity of the IMM based model, in this experiment, the CV-CA movement obeys a random walk. The initializing speed of CV movement model in the first second is $1.5 \mathrm{~m} / \mathrm{s}$, and in the CA part, the acceleration of $a=0.2 \mathrm{~m} / \mathrm{s}^{2}$ lasts for 0.5 second, and then acceleration changes to $-0.1 \mathrm{~m} / \mathrm{s}^{2}$. The tracking trajectories are given in Figure 20 and the distance errors for each filter are plotted in Figure 21. It is concluded that single model based approach cannot fully track the target, leading to a large error.

The last tracking task is concerned with the multitarget case, depicted in Figure 22. In this scenario, two targets are assumed to move in opposite directions. First, the target $\mathcal{A}$ moves from $(1.0,1.3) \mathrm{m}$ to $(1.65,0.92) \mathrm{m}$, whereas 


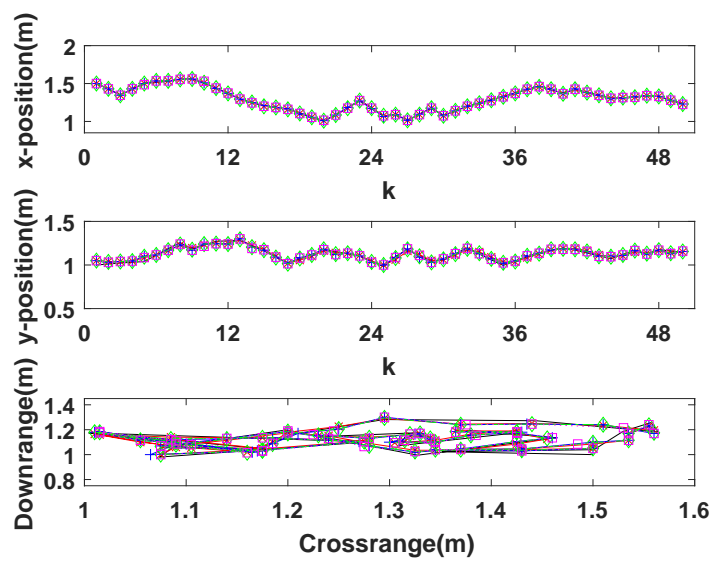

Fig. 20: Outputs of the tracker and the ground truth in the random walk with CV-CA movement. The top row indicates the target position along $x$. The middle row indicates the target position along $y$. The bottom row indicates the real trajectory. '-': real trajectory; + : PF with CV; $\times$ : KF with CV; $\square$ : IMM-PF; $\diamond:$ IMM-KF.

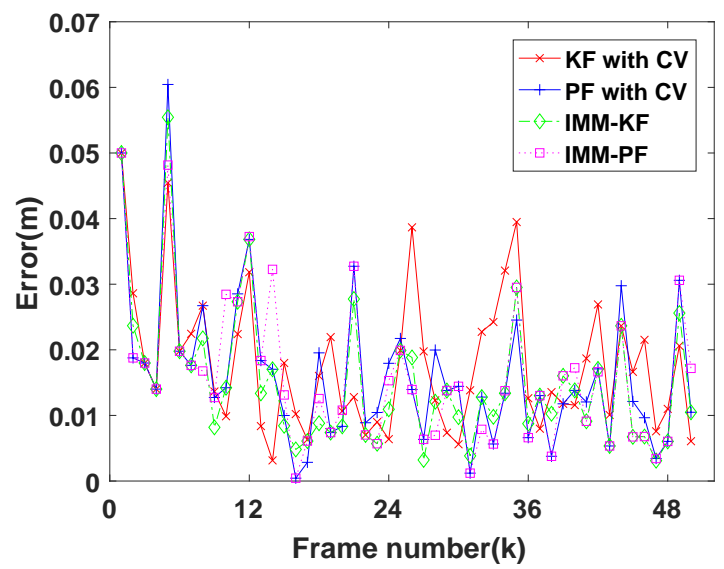

Fig. 21: Distance errors for each filter under CV-CA movement with random walk.

the target $\mathcal{B}$ moves from $(1.8,1.3) \mathrm{m}$ to $(1.15,0.92) \mathrm{m}$. Finally, two targets both move away from the radar at the same time. In the multitarget scenario, the biggest challenge for the tracking task lies when two targets intersects. To be specific, the interactions are expected to produce ghosting phenomenon in the TWR imaging, which results in an increased false alarms [40]. When two targets are too close, they will be detected as one target in the imaging. However, in our case, since the observation windows are not deleted, both targets are still correctly tracked. In addition, to demonstrate the case when a target disappears and reappears, after the above movements, we keep the target $\mathcal{B}$ still and the target $\mathcal{A}$ starts a random walk. In the next second, target $\mathcal{B}$ restarts. The outputs of trackers are provided in Figure 23. Figure 24 shows the estimation errors for each model. It is evident from the results that even under the influence of the target-to-target interactions, the results of trackers along $x$ are still identical to the true values, which shows the robustness of the proposed method. The estimated positions along $y$ are also in a good agreement with the ground truth. From the top row of Fig. 23, it is seen that the target $\mathcal{B}$ disappeared after the 
11th frame, and the proposed tracking strategy terminates the corresponding tracker after the 13th frames. At the beginning of the next second, the 21 st frame to be exact, target $\mathcal{B}$ is re-captured and assigned a new observation window and trackers.

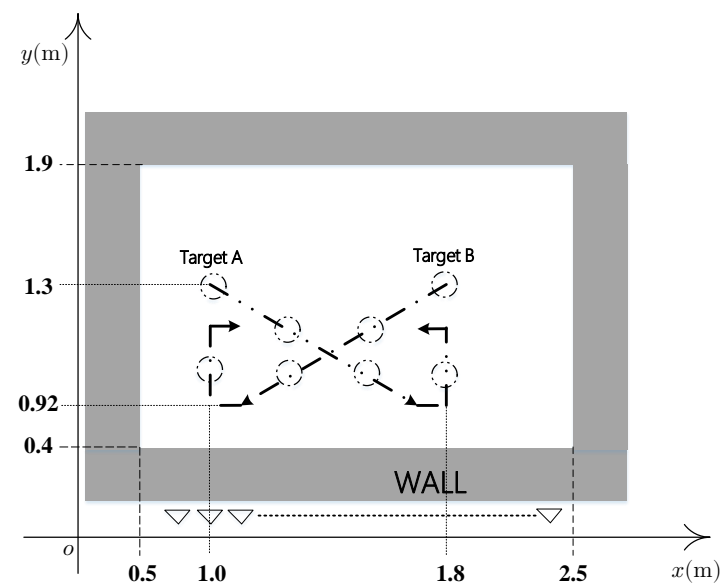

Fig. 22: Two target scenario: The dashed dot circles depict the target position.

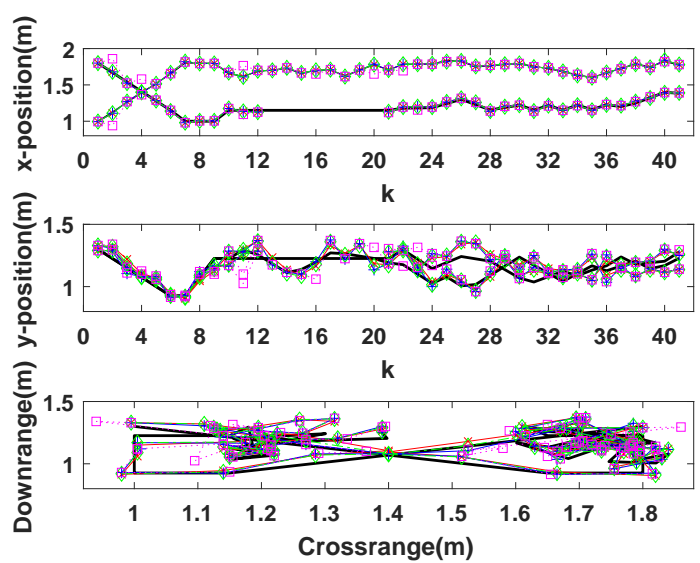

Fig. 23: Outputs of the tracker and the ground truth in the multitarget scenario. The top row indicates the target position along $x$. The middle row indicates the target position along $y$. The bottom row indicates the real trajectories. '-': real trajectory; +: PF with $\mathrm{CV}$; $\times$ : KF with $\mathrm{CV}$; $\square$ : IMM-PF; $\diamond:$ IMM-KF.

Finally, to quantitatively evaluate the tracking performance, the tracker accuracies in the above experiments are provided in Table V. The estimation error between estimated and true value is denoted by $\delta_{p}$, which is defined as

$$
\delta_{p}=\sqrt{\left(p_{x}-\hat{p}_{x}\right)^{2}+\left(p_{y}-\hat{p}_{y}\right)^{2}}
$$

where $p_{x}$ and $p_{y}$ are true positions, and $\hat{p}_{x}$ and $\hat{p}_{y}$ are estimated positions. As discussed previously, the trackers showed the best performance in the second task. In general, the IMM-based filters have better accuracy than KF/PF with the single $\mathrm{CV}$ model, but the estimation errors are all in the range $2 \mathrm{~cm}-8 \mathrm{~cm}$, which is consistent with the recent studies [27], [28], [39], [41]. 


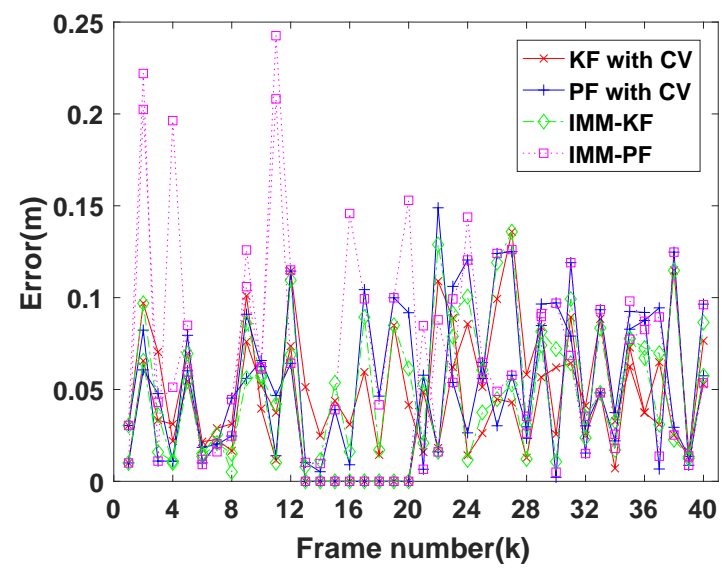

Fig. 24: Distance errors for each filter under multitarget case.

TABLE V: Tracker accuracy for the tracking tasks

\begin{tabular}{|c|c|c|c|}
\hline Task & Target & Tracker & Accuracy $\delta_{p}(\mathrm{~m})$ \\
\hline \multirow{4}{*}{ Semicircular task } & \multirow{4}{*}{ Target $\mathcal{A}$} & KF with $\mathrm{CV}$ & 0.0296 \\
\hline & & PF with CV & 0.0292 \\
\hline & & IMM-KF & 0.0307 \\
\hline & & IMM-PF & 0.0329 \\
\hline \multirow{4}{*}{ Random-walk task } & \multirow{4}{*}{ Target $\mathcal{A}$} & KF with CV & 0.0217 \\
\hline & & PF with CV & 0.0220 \\
\hline & & IMM-KF & 0.0217 \\
\hline & & IMM-PF & 0.0226 \\
\hline \multirow{4}{*}{ CV-CA-CV task } & \multirow{4}{*}{ Target $\mathcal{A}$} & $\mathrm{KF}$ with $\mathrm{CV}$ & 0.0140 \\
\hline & & PF with CV & 0.0166 \\
\hline & & IMM-KF & 0.0140 \\
\hline & & IMM-PF & 0.0150 \\
\hline \multirow{4}{*}{ CV-CA task } & \multirow{4}{*}{ Target $\mathcal{A}$} & KF with $\mathrm{CV}$ & 0.0178 \\
\hline & & PF with CV & 0.0161 \\
\hline & & IMM-KF & 0.0151 \\
\hline & & IMM-PF & 0.0157 \\
\hline \multirow{8}{*}{ Multitarget task } & \multirow{4}{*}{ Target $\mathcal{A}$} & KF with CV & 0.0427 \\
\hline & & PF with CV & 0.0511 \\
\hline & & IMM-KF & 0.0455 \\
\hline & & IMM-PF & 0.0650 \\
\hline & \multirow{4}{*}{ Target $\mathcal{B}$} & KF with CV & 0.0508 \\
\hline & & PF with CV & 0.0513 \\
\hline & & IMM-KF & 0.0486 \\
\hline & & IMM-PF & 0.0765 \\
\hline
\end{tabular}




\section{CONCLUSION}

To obtain the trajectory of the target, in this study, two essential components of clutter reduction and target tracking are developed, using TWR system. For the clutter reduction, the data suggests a compelling evidence that the clutter returns are of low-rank. Based on this, a joint low-rank and sparse framework is utilized to simultaneously remove the clutter and form the image. To effectively capture the target, a simple observation window search method is developed, and finally, the tracking is conducted based on $\mathrm{KF} / \mathrm{PF}$, where IMM technique is explored to combine different movement models. The experiments of clutter reduction indicate that the thickness of the wall plays a more important role than the permittivity. The experiments of target tracking show that IMM based model performs better than that of single model in the case of complex motions.

\section{ACKNOWLEDGMENT}

This work was jointly supported by the National Natural Science Foundation of China under Grants 61501072 and 61801066.

\section{REFERENCES}

[1] M. Amin, Through-the-wall Radar Imaging, CRC Press, Boca Raton, FL, USA, 2010.

[2] M. Amin and K. Sarabandi, "Special issue on remote sensing of building interior," IEEE Transactions on Geoscience and Remote Sensing, vol. 47, no. 5, pp. 1267-1268, May 2009.

[3] C. Lai and R. M. Narayanan, "Ultrawideband random noise radar design for through-wall surveillance," IEEE Transactions on Aerospace and Electronic Systems, vol. 46, no. 4, pp. 1716-1730, Oct 2010.

[4] L.-P. Song, C. Yu, and Q. H. Liu, “Through-wall imaging (twi) by radar: 2-d tomographic results and analyses,” IEEE Transactions on Geoscience and Remote Sensing, vol. 43, no. 12, pp. 2793-2798, Dec 2005.

[5] R. Chandra, A.N. Gaikwad, D. Singh, and M. J. Nigam, "An approach to remove the clutter and detect the target for ultra-wideband through-wall imaging," Journal of Geophysics and Engineering, vol. 5, no. 4, pp. 412-419, 102008.

[6] P. K. Verma, A. N. Gaikwad, D. Singh, and M. J. Nigam, "Analysis of clutter reduction techniques for through wall imaging in uwb range," Progress In Electromagnetics Research, vol. 17, pp. 29-48, 2009.

[7] F. H. C. Tivive, A. Bouzerdoum, and M. G. Amin, "An svd-based approach for mitigating wall reflections in through-the-wall radar imaging," in 2011 IEEE RadarCon (RADAR), May 2011, pp. 519-524.

[8] F. Ahmad, J. Qian, and M. G. Amin, "Wall clutter mitigation using discrete prolate spheroidal sequences for sparse reconstruction of indoor stationary scenes," IEEE Transactions on Geoscience and Remote Sensing, vol. 53, no. 3, pp. 1549-1557, March 2015.

[9] Z. Zhu and M. B. Wakin, "On the dimensionality of wall and target return subspaces in through-the-wall radar imaging," in 2016 4th International Workshop on Compressed Sensing Theory and its Applications to Radar, Sonar and Remote Sensing (CoSeRa), Sep. 2016, pp. 110-114.

[10] V. H. Tang, A. Bouzerdoum, S. L. Phung, and F. H. C. Tivive, "Radar imaging of stationary indoor targets using joint low-rank and sparsity constraints," in 2016 IEEE International Conference on Acoustics, Speech and Signal Processing (ICASSP), March 2016, pp. $1412-1416$. 
[11] A. Bouzerdoum, V. H. Tang, and S. L. Phung, "A low-rank and jointly-sparse approach for multipolarization through-wall radar imaging," in 2017 IEEE Radar Conference (RadarConf), May 2017, pp. 0263-0268.

[12] V. H. Tang, A. Bouzerdoum, and S. L. Phung, "Multipolarization through-wall radar imaging using low-rank and jointly-sparse representations," IEEE Transactions on Image Processing, vol. 27, no. 4, pp. 1763-1776, April 2018.

[13] H. Q. Liu, D. Li, Y. Zhou, and T.-K. Truong, "Simultaneous radio frequency and wideband interference suppression in SAR signals via sparsity exploitation in time-frequency domain," IEEE Trans. Geoscience and Remote Sensing, vol. 56, no. 10, pp. 5780-5793, Oct. 2018.

[14] D. Li, M. Zhan, H. Liu, Y. Liao, and G. Liao, "A robust translational motion compensation method for isar imaging based on keystone transform and fractional fourier transform under low snr environment," IEEE Transactions on Aerospace and Electronic Systems, vol. 53, no. 5, pp. 2140-2156, Oct 2017.

[15] Y. Zhang and T. Xia, "In-wall clutter suppression based on low-rank and sparse representation for through-the-wall radar," IEEE Geoscience and Remote Sensing Letters, vol. 13, no. 5, pp. 671-675, May 2016.

[16] D. Li, M. Zhan, J. Su, H. Liu, X. Zhang, and G. Liao, "Performances analysis of coherently integrated cpf for lfm signal under low snr and its application to ground moving target imaging," IEEE Transactions on Geoscience and Remote Sensing, vol. 55, no. 11, pp. 6402-6419, Nov 2017.

[17] L. Qiu, T. Jin, Y. He, B. Lu, and Z. Zhou, "Sparse and low-rank matrix decomposition based micro-motion target indication in through-the-wall radar," Electronics Letters, vol. 53, no. 3, pp. 191-192, 2017.

[18] S. R. Becker, E. J. Candès, and M. C. Grant, "Templates for convex cone problems with applications to sparse signal recovery," Mathematical Programming Computation, vol. 3, no. 3, pp. 165, Jul 2011.

[19] M. G. Amin and F. Ahmad, "Change detection analysis of humans moving behind walls," IEEE Transactions on Aerospace and Electronic Systems, vol. 49, no. 3, pp. 1410-1425, July 2013.

[20] A. F. Martone, K. Ranney, and C. Le, "Noncoherent approach for through-the-wall moving target indication," IEEE Transactions on Aerospace and Electronic Systems, vol. 50, no. 1, pp. 193-206, January 2014.

[21] Y. Bar-Shalom, P. Willett, and X. Tian, Tracking and Data Fusion A Handbook of Algorithms, YBS publishing Storrs, CT, USA:, 2011.

[22] Y. Bar-Shalom, F. Daum, and J. Huang, "The probabilistic data association filter," IEEE Control Systems Magazine, vol. 29, no. 6, pp. 82-100, Dec 2009.

[23] B. Vo, B. Vo, and A. Cantoni, "Bayesian filtering with random finite set observations," IEEE Transactions on Signal Processing, vol. 56, no. 4, pp. 1313-1326, April 2008.

[24] P. Braca, S. Marano, V. Matta, and P. Willett, "Asymptotic efficiency of the phd in multitarget/multisensor estimation," IEEE Journal of Selected Topics in Signal Processing, vol. 7, no. 3, pp. 553-564, June 2013.

[25] E. Mazor, A. Averbuch, Y. Bar-Shalom, and J. Dayan, "Interacting multiple model methods in target tracking: a survey," IEEE Transactions on Aerospace and Electronic Systems, vol. 34, no. 1, pp. 103-123, Jan 1998.

[26] K. Jo, K. Chu, K. Lee, and M. Sunwoo, "Integration of multiple vehicle models with an imm filter for vehicle localization," in 2010 IEEE Intelligent Vehicles Symposium, June 2010, pp. 746-751.

[27] Y. He, T. Savelyev, and A. Yarovoy, "Two-stage algorithm for extended target tracking by multistatic uwb radar," in Proceedings of 2011 IEEE CIE International Conference on Radar, Oct 2011, vol. 1, pp. 795-799.

[28] X. Chen, H. Leung, and M. Tian, "Multitarget detection and tracking for through-the-wall radars," IEEE Transactions on Aerospace and Electronic Systems, vol. 50, no. 2, pp. 1403-1415, April 2014.

[29] F. Ahmad, G. J. Frazer, S. A. Kassam, and M. G. Amin, "Design and implementation of near-field, wideband synthetic aperture beamformers," IEEE Transactions on Aerospace and Electronic Systems, vol. 40, no. 1, pp. 206-220, Jan 2004. 
[30] S. Boyd, N. Parikh, E. Chu, B. Peleato, and J. Eckstein, "Distributed optimization and statistical learning via the alternating direction method of multipliers," Found. Trends Mach. Learn., vol. 3, no. 1, pp. 1-122, Jan. 2011.

[31] Y. Xie, S. Gu, Y. Liu, W. Zuo, W. Zhang, and L. Zhang, "Weighted schatten $p$-norm minimization for image denoising and background subtraction," IEEE Transactions on Image Processing, vol. 25, no. 10, pp. 4842-4857, Oct 2016.

[32] R. Mazumder, T. Hastie, and R. Tibshirani, "Spectral regularization algorithms for learning large incomplete matrices," Journal of machine learning research, vol. 11, no. Aug, pp. 2287-2322, 2010.

[33] G. Wang and M. G. Amin, "Imaging through unknown walls using different standoff distances," IEEE Transactions on Signal Processing, vol. 54, no. 10, pp. 4015-4025, Oct 2006.

[34] G. Wang, M. G. Amin, and Y. Zhang, "New approach for target locations in the presence of wall ambiguities," IEEE Transactions on Aerospace and Electronic Systems, vol. 42, no. 1, pp. 301-315, Jan 2006.

[35] M. S. Grewal, Kalman Filtering: Theory and Practice using MATLAB, New York : John Wiley, 2001.

[36] B. Ristic, A. Arulampalam, and N. Gordon, Beyond the Kalman Filter-Particle Filters for Tracking Applications, Artech House, Boston, 2004.

[37] C. Warren, A. Giannopoulos, and I. Giannakis, "gprmax: Open source software to simulate electromagnetic wave propagation for ground penetrating radar," Computer Physics Communications, vol. 209, pp. 163 - 170, 2016.

[38] C. Gabriel, S. Gabriel, and E. Corthout, "The dielectric properties of biological tissues: I. literature survey," Physics in Medicine and Biology, vol. 41, no. 11, pp. 2231-2249, nov 1996.

[39] G. Gennarelli, G. Vivone, P. Braca, F. Soldovieri, and M. G. Amin, "Multiple extended target tracking for through-wall radars," IEEE Transactions on Geoscience and Remote Sensing, vol. 53, no. 12, pp. 6482-6494, Dec 2015.

[40] G. Gennarelli and F. Soldovieri, "Multipath ghosts in radar imaging: Physical insight and mitigation strategies," IEEE Journal of Selected Topics in Applied Earth Observations and Remote Sensing, vol. 8, no. 3, pp. 1078-1086, March 2015.

[41] J. Rovnakova and D. Kocur, "Compensation of wall effect for through wall tracking of moving targets," Radioengineering, vol. 18, no. 2, pp. 189-195, 2009. 